\title{
Turbulence characteristics in sharp open-channel bends
}

\author{
K. Blanckaert ${ }^{\text {a) }}$ \\ ICARE-ENAC, Ecole Polytechnique Fédérale, CH-1015 Lausanne, Switzerland \\ H. J. de Vriend \\ Delft University of Technology, P.O. Box 5048, 2600 GA Delft, The Netherlands
}

(Received 24 March 2004; accepted 25 January 2005; published online 11 April 2005)

\begin{abstract}
In spite of its importance, little is known about the turbulence characteristics in open-channel bends. This paper reports on an experimental investigation of turbulence in one cross section of an open-channel bend. Typical flow features are a bicellular pattern of cross-stream circulation (secondary flow) and a turbulence activity in the outer bend that is significantly less than in the equivalent straight uniform shear flow. Measured distributions are given of the turbulent kinetic energy, its production, the mixing coefficients, some parameters characterizing the turbulence structure, and the fourth-order correlations of the turbulent velocity fluctuations. The transport equation for the turbulent kinetic energy is evaluated term by term, on the basis of the measured data. The results show that the turbulence structure is different from straight uniform flow, in that the Reynolds stress tensor is more diagonally dominant. This is shown to be the main cause of the observed reduction of turbulence activity in the outer bend. The usual two-equation turbulence closure models include a transport equation for the turbulent kinetic energy, but they do not account for this modified turbulence structure. The departures of the measured turbulence structure from its equivalent in straight uniform shear flow are related to a curvature-flux-Richardson number $R_{f}$ which includes the streamline curvature. Such a relation may be useful to improve simple turbulence closure models for curved open-channel flow. (C) 2005 American Institute of Physics.
\end{abstract}

[DOI: $10.1063 / 1.1886726]$

\section{INTRODUCTION}

Turbulence plays an important role in open-channel flows. It is to a large extent responsible for the spreading and mixing of heat and dissolved or suspended matter (sediments, pollutants, oxygen, etc.). It also has a strong interaction with the mean velocity field and the boundary shear stress. Turbulence plays an important role in the formation of cross-stream circulation cells, ${ }^{1}$ which in their turn influence the distribution of the velocity and the boundary shear stress. $^{2}$ This interaction is reflected by the sensitivity of flow models to the turbulence closure.

Open-channel bends are ubiquitous in alluvial lowland rivers, but they also occur in mountain rivers and man-made channels. They influence the conveyance capacity of the channel, may lead to undesired erosion/deposition and enhanced mixing, and provide a suitable place for the intake of relatively clear water. From an ecological point of view, meanders give rise to a spatial variation of flow conditions, thus providing a range of habitats. Meander migration is an important agent in floodplain rejuvenation. Many good reasons, therefore, to investigate curved open-channel flow.

In spite of the relevance of both turbulence and bends, little is known about the turbulence characteristics in openchannel bends. The study of environmental problems in bends, such as spreading and mixing processes, suffers from an almost complete lack of experimental data on turbulence. Numerical simulations of the flow in open-channel bends

a)Telephone: +41/21/6932378. Electronic mail: koen.blanckaert@epfl.ch frequently use extensions of turbulence closures that were developed for rectilinear shear flow. They often give poor results, due to the different turbulence structure. The lack of experimental data, especially from simultaneous highresolution measurements of all three velocity components, hampers the development of improved turbulence models.

The main goal of this paper is to present detailed experimental data on the turbulence characteristics in an openchannel bend, including distributions of the turbulent kinetic energy (tke), the production of tke, the mixing coefficients, parameters characterizing the turbulence structure, and fourth-order turbulence correlations. These experimental data are measured in one cross section of a bend, in which the flow is characterized by the existence of a bicellular pattern of cross-stream circulation (secondary flow) and by a significant reduction of the turbulence activity in the outer bend as compared to straight uniform shear flow.

Another goal of this paper is to analyze the mechanisms that lead to the reduction of the turbulence activity in the outer bend, via a term-by-term evaluation of the transport equation for the turbulent kinetic energy on the basis of the measured data. Special attention is given to the terms representing the production of tke and to those representing its advective transport by the cross-stream motion.

A third goal of this paper is to investigate whether turbulence closure models can be improved by means of ad hoc modifications that account for the influence of streamline curvature. 


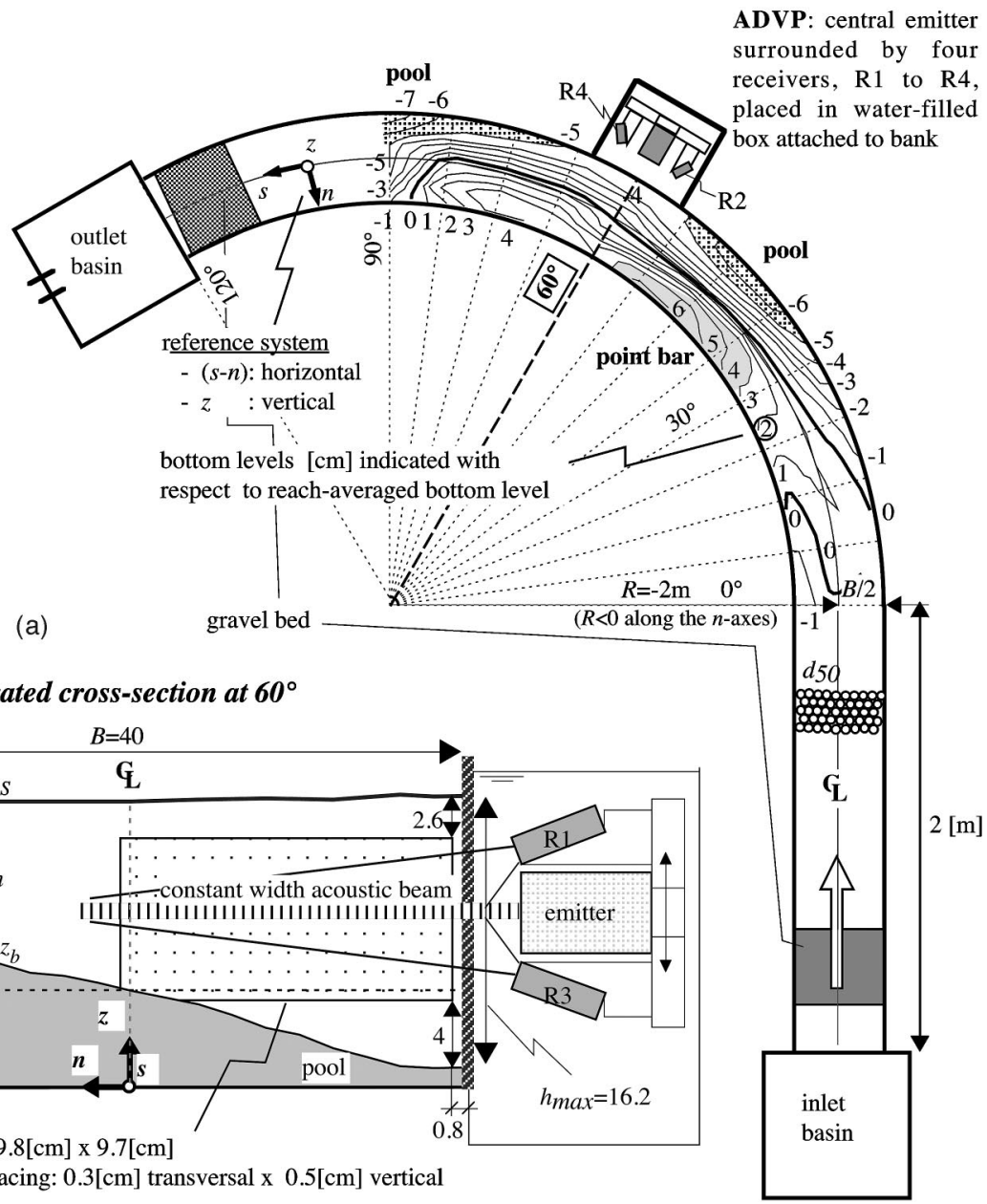

FIG. 1. (a) Experimental setup, bed topography, and reference system; (b) measuring grid in the cross section at $60^{\circ}$, acoustic Doppler velocity profiler (ADVP) configuration.

\section{THE EXPERIMENT}

Natural river bends occur under a wide variety of spatial scales, planform shapes, and hydraulic parameters. Instead of attempting to design an experiment that is representative of meandering natural rivers, we have opted for a sharp singlebend configuration consisting of a straight inflow reach followed by a constant curvature bend. The aim was to isolate and emphasize curvature effects and minimize contamination by upstream geometrical and hydraulic effects. By considering a relatively sharp bend, the curvature effects are more pronounced, hence better visible.

Flow measurements were carried out in a laboratory flume which width $B$ is $0.4 \mathrm{~m}$ and consists of a $2 \mathrm{~m}$ long straight approach reach, followed by a $120^{\circ}$ bend with a constant radius of curvature $R$ of $2 \mathrm{~m}$ at the centerline.

The bed, which was covered by a nearly uniform quartz sand with median grain size diameter $d_{50}=2.1 \mathrm{~mm}$, was in equilibrium with the flow. In a preliminary run, the initially flat sand bed was deformed by the flow under clear-waterscour conditions, i.e., with the bed shear stress in the straight inflow reach at or just below the critical shear stress for sediment motion. This preliminary run led to the formation of a typical bar-pool topography in the bend (Fig. 1), which shows important spatial variations but is stable (zero time derivative) without active sediment transport.

The hydraulic conditions of the flow are shown in Table I. The parameters $R / B=5$ and $R / H=17.9$, and hence the aspect ratio $B / H=3.6$, correspond to a bend that is rather sharp and significantly narrower than usual in natural lowland rivers. These ratios do occur, however, in mountain rivers and man-made channels. Moreover, the flow in a wide bend with a mobile-bed topography is concentrated mostly in the outer part of the cross section, where the depth is significantly larger than in the shallow inner bend with its almost "deadwater" zone. ${ }^{3,4}$ The "effective" aspect ratio there is significantly smaller than in a straight reach of the same channel. The flow in the outer bend in the present experiment is therefore thought to be rather similar to the flow in the deepest part of sharp natural channel bends. Although similarities exist, it should be borne in mind that no experimental setup can be representative of natural rivers in general, whose turbulence characteristics may significantly depend on hydraulic parameters such as the aspect ratio $B / H$, or the curvature ratio $R / H$.

Nonintrusive velocity measurements were made on a fine grid in the outer half of the cross section at $60^{\circ}$ from the 
TABLE I. Hydraulic conditions.

\begin{tabular}{|c|c|c|c|c|c|c|c|c|c|c|c|c|c|}
\hline$R^{\mathrm{a}}[\mathrm{m}]$ & $B^{\mathrm{b}}[\mathrm{m}]$ & $d_{50}{ }^{\mathrm{c}}[\mathrm{mm}]$ & $Q^{\mathrm{d}}[1 / \mathrm{s}]$ & $H^{\mathrm{e}}[\mathrm{m}]$ & $S_{s}{ }^{\mathrm{f}}[\%]$ & $U^{\mathrm{g}}[\mathrm{m} / \mathrm{s}]$ & $C_{f}^{\mathrm{h}}$ & $\operatorname{Re}^{\mathrm{i}}\left[10^{3}\right]$ & $\operatorname{Re}_{*}{ }^{\mathrm{j}}$ & $\mathrm{Fr}^{\mathrm{k}}$ & $R / B$ & $R / H$ & $B / H$ \\
\hline-2.0 & 0.40 & 2.1 & 17 & 0.11 & 0.189 & 0.38 & 0.008 & 42 & 70 & 0.36 & 5 & 17.9 & 3.6 \\
\hline
\end{tabular}

${ }^{\mathrm{a}}$ Centerline radius of curvature (negative along the $n$ axis).

${ }^{\mathrm{b}}$ Channel width.

${ }^{c}$ Median grain size diameter of the bed material.

${ }^{\mathrm{d}}$ Flow discharge.

'Overall mean flow depth $\approx$ depth at centerline.

${ }^{\mathrm{f}}$ Overall mean water-surface slope at the centerline.

${ }^{\mathrm{g}} U=Q /(B H)$ : overall mean velocity.

${ }^{\mathrm{h}} C_{f}=\left(u_{*} / U\right)^{2}$ : friction factor (estimated by Blanckaert and Graf (Ref. 5), $\tau_{b}$, bottom shear stress; $\tau_{b} / \rho=u_{*}^{2}=C_{f} U^{2}$.

${ }^{\mathrm{i}} \mathrm{Re}=U H / \nu$ : overall mean flow Reynolds number.

${ }^{\mathrm{j}} \mathrm{Re}_{*}=u_{*} k_{s} / \nu$ : overall mean particle Reynolds number, $\nu$, molecular viscosity; $k_{s}$, Nikuradse equivalent sand roughness.

${ }^{\mathrm{k}} \mathrm{Fr}=U /(g H)^{1 / 2}$ : overall mean Froude number.

bend entrance [Fig. 1(b)]. By imposing physical boundary conditions (no slip condition, free shear, etc.), some experimental data have been extrapolated into the zones close to the water surface and the bed, outside the measuring grid, as explained in detail in Blanckaert and Graf. ${ }^{5}$ These extrapolations, however, are not essential for the purpose of the present analysis. The measured data are represented and analyzed in a coordinate system with the $s$ axis along the channel centerline, the $n$ axis perpendicular to it and pointing to the left and the $z$ axis vertically upwards, perpendicular to the horizontal $(s, n)$ plane (Fig. 1).

The velocity measurements were made with an acoustic Doppler velocity profiler (ADVP), developed at EPFL. ${ }^{6}$ The ADVP was mounted in a water-filled box attached to the outside of the wall [Figs. 1(a) and 1(b)] and measured the three instantaneous velocity components $v_{j}(t)$ simultaneously along the main axis of the measuring device. Such transversal profiles, extending over half the channel width, were measured at vertical intervals of $0.5 \mathrm{~cm}$ [Fig. 1(b)]. The sampling frequency was $44.6 \mathrm{~Hz}$ and the acquisition time was $180 \mathrm{~s}$. This provides sufficient information to derive the mean velocity vector $v=\left(v_{s}, v_{n}, v_{z}\right)$, as well as the fluctuating velocity vector $v^{\prime}=\left(v_{s}^{\prime}, v_{n}^{\prime}, v_{z}^{\prime}\right)$ and all turbulent correlations $\overline{v_{i}^{\prime a} v_{j}^{\prime b}}(i, j=s, n, z ; a$ and $b$ are integers, an overbar denotes a time-averaged value).

A detailed description of the experimental setup, the data-processing procedures and the measuring grid is given in Blanckaert and Graf. ${ }^{5}$ More information on the working principle of the ADVP, its experimental uncertainty, and its comparison with other velocity meters can be found in Rolland, ${ }_{6}^{7}$ Lemmin and Rolland, ${ }^{8}$ Hurther and Lemmin, ${ }^{9,10}$ Hurther, ${ }^{6}$ Blanckaert and Graf, ${ }^{5}$ Blanckaert and Lemmin ${ }^{11}$ and Blanckaert and de Vriend. ${ }^{1}$ In summary, the uncertainty is estimated at less than $4 \%$ in the mean velocities, and at less than $10 \%$ in the turbulent normal stresses. The uncertainty in the turbulent shear stresses is slightly less than in the turbulent normal stresses. For the present data set, Blanckaert and $\mathrm{Graf}^{5}$ have made more conservative estimates for the turbulent shear stresses, $\pm 20 \%$, and for the cross-stream velocities, $\pm 0.002 \mathrm{~m} / \mathrm{s}$ (which amounts to about $\pm 10 \%$ ). These higher relative uncertainties are mainly due to the low mean values of these quantities. The uncertainty in the turbulence measurements increases progressively towards fixed boundaries, due to the steep mean velocity gradient in the measuring volume. The lower $20 \%$ of the boundary layer is affected by it. For that reason, the ADVP measurements focus on the outer-flow region, away from the boundaries.

These uncertainties mainly concern statistical errors; systematic errors, due to instrument misalignment for example, are much smaller.

To eliminate the experimental scatter, the raw experimental data have been fitted using two-dimensional smoothing splines with weight functions. ${ }^{12}$ This procedure allows using relatively short acquisition times (180 s). Furthermore, it allows for a more precise evaluation of the terms in the transport equation for the tke, including derivatives and/or products of measured quantities. This smoothing technique is illustrated in Blanckaert and Graf. ${ }^{5}$ According to the procedure outlined by Blanckaert and de Vriend, ${ }^{1}$ the uncertainty in these evaluated terms is estimated at less than $40 \%$. This is sufficient for the purpose of this paper, since our interpretations focus on first-order effects.

\section{EXPERIMENTAL RESULTS}

Presentations of the distributions of the mean velocities and the turbulent stresses $\overline{v_{i}^{\prime} v_{j}^{\prime}}$ have been reported by Blanckaert and Graf. ${ }^{5}$ Only the measured data that are of particular relevance to this paper are briefly presented in this section.

The flow field in the investigated cross section at $60^{\circ}$ is characterized by a bicellular pattern of cross-stream motion $\left(v_{n}, v_{z}\right)$, as shown in Fig. 2. The classical helical motion, termed center-region cell, is discernable in the central part of the cross section. The cross-stream velocities involved are typically $10 \%$ of the mean downstream velocity. In the upper part of the outer-bank region, a weaker counterrotating cell occurs, with cross-stream velocities of typically $3 \%$ of the mean downstream velocity. The mechanisms leading to these two circulation cells have been analyzed by Blanckaert and de Vriend. ${ }^{1-13}$

Figures 3(a) and 3(b) show the normalized distributions of the mean-flow kinetic energy $K /\left(1 / 2 U^{2}\right)$, and the turbulent kinetic energy $k /\left(1 / 2 u_{*, 60}^{2}\right)$, per unit mass, in which $K$ and $k$ are defined as

$$
K=\frac{1}{2}\left(v_{s}^{2}+v_{n}^{2}+v_{z}^{2}\right) \approx \frac{1}{2} v_{s}^{2}, \quad k=\frac{1}{2}\left(\overline{v_{s}^{\prime 2}}+\overline{v_{n}^{\prime 2}}+\overline{v_{z}^{\prime 2}}\right) .
$$

The characteristic shear velocity, $u_{*, 60}=\sqrt{g R_{h}\left(-\partial z_{S, 60} / \partial s\right)}$ $=0.045 \mathrm{~m} / \mathrm{s}$, is based on the downstream water-surface gra- 


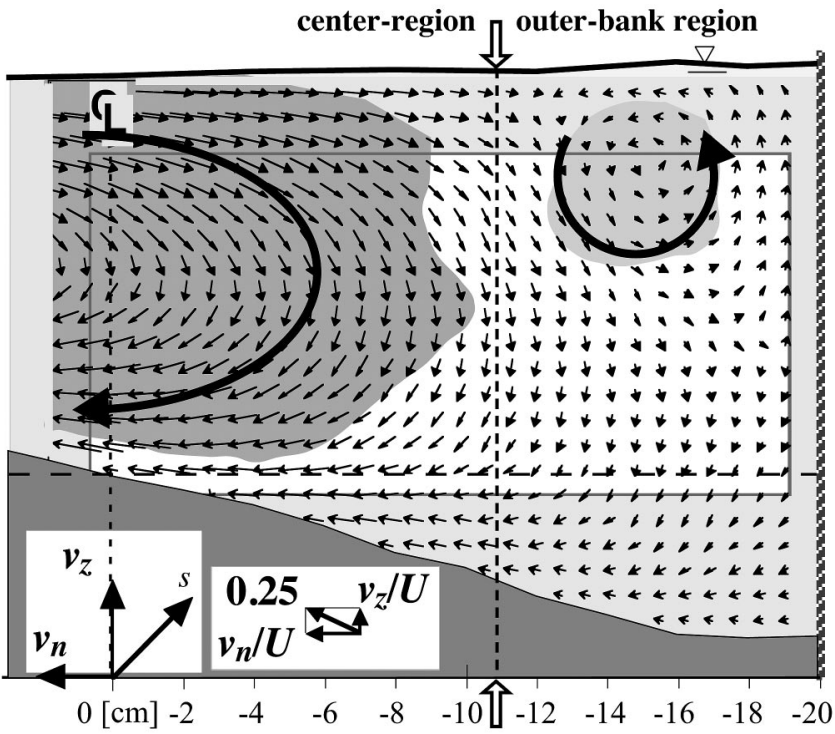

FIG. 2. Vector representation of normalized cross-stream motion $\left(v_{n}, v_{z}\right) / U$.

dient at the centerline, $-\partial z_{s, 60} / \partial s=0.289 \%$, and the hydraulic radius, $R_{h}=0.07 \mathrm{~m}$, in the section at $60^{\circ}$.

In straight uniform flow, the vertical profiles of $K_{\text {straight }}$ $=v_{s, \text { straight }}^{2} / 2$ typically increase from zero at the bed to a maximum value near the water surface, whereas the vertical profiles of $k_{\text {straight }}$ typically decrease monotonically from the bed towards the water surface. Assuming a logarithmic downstream velocity and an exponentially decreasing tke from the bed towards the water surface (Nezu and Nakagawa ${ }^{14}$ )

$$
\begin{aligned}
& v_{s, \text { straight }}=U_{s}\left[1+\kappa^{-1} \sqrt{C_{f}}(1+\ln z / h)\right]=U_{s} f_{s}, \\
& k_{\text {straight }}=4.78 u_{*}^{2} e^{-2(z / h)},
\end{aligned}
$$

it is found by integration of Eqs. (2) and (3) over the flow depth $h$ that $\langle k\rangle /\langle K\rangle$ uniquely depends on the friction factor:

$$
\begin{aligned}
\left.\frac{\langle k\rangle}{\langle K\rangle}\right|_{\text {straight }} & =\frac{\left\langle k_{\text {straight }}\right\rangle}{\frac{1}{2}\left\langle v_{s, \text { straight }}^{2}\right\rangle}=\frac{4.133}{\left\langle f_{s}^{2}\right\rangle}\left(\frac{u_{*}}{U_{s}}\right)^{2}=\frac{4.133}{\left\langle f_{s}^{2}\right\rangle} C_{f} \\
& \approx 4.1 C_{f},
\end{aligned}
$$

where $f_{s}$ is the form of the vertical profile of the downstream velocity $v_{s}, \kappa$ is the Karman constant, \langle\rangle indicate local depth-averaged values and $U_{s}=\left\langle v_{\mathrm{s}}\right\rangle$. The overall resistance factor $C_{f}$ in the experiments was about 0.008 , which means that $\langle k\rangle /\langle K\rangle \approx 0.03$ is to be expected for straight flow.

In our curved-flow experiment the distributions of $K$ and $k$ show a more complex behavior. The vertical profiles of $K /\left(1 / 2 U^{2}\right) \approx\left(v_{s} / U\right)^{2}$ [Fig. 3(a)] do not increase from the bed to the surface but have their maximum in the lower part of the water column. $K /\left(1 / 2 U^{2}\right)$ increases from the centerline in outward direction to reach a maximum of about 2.2 at the separation between the circulation cells. The mass is conserved, the large velocities are driven to the outside, hence

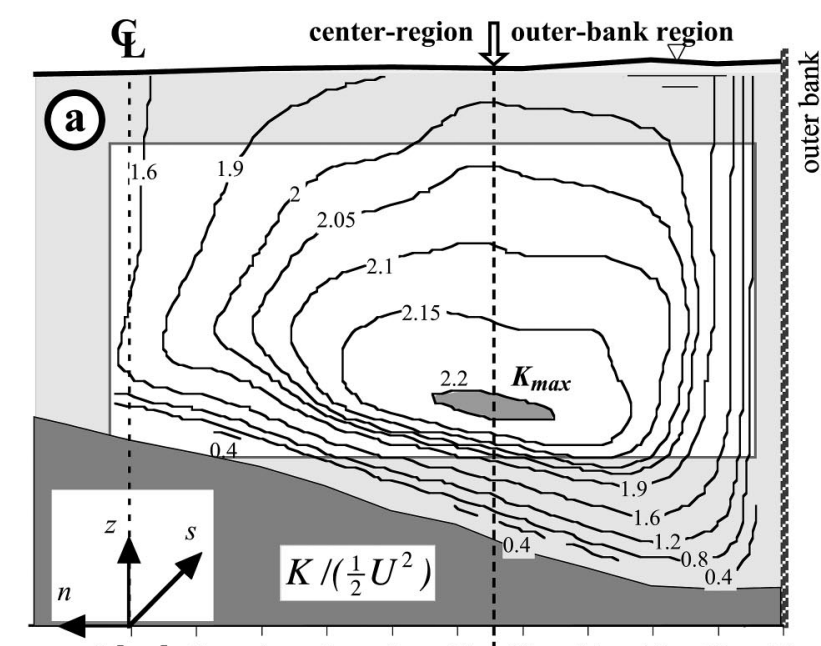

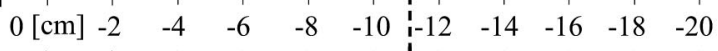

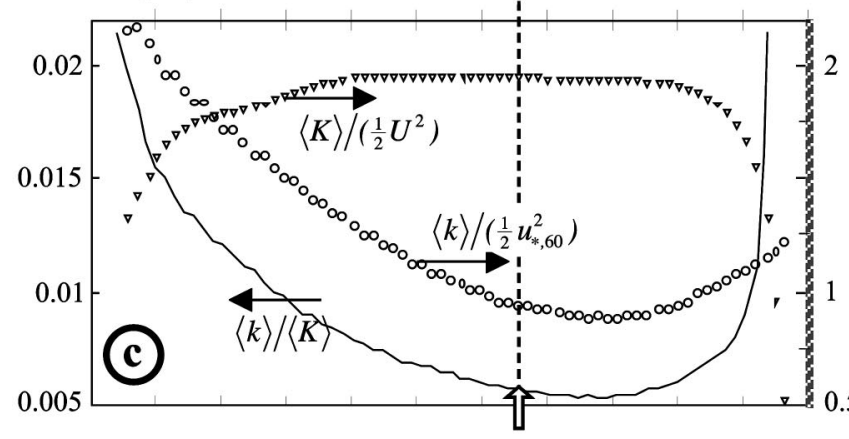

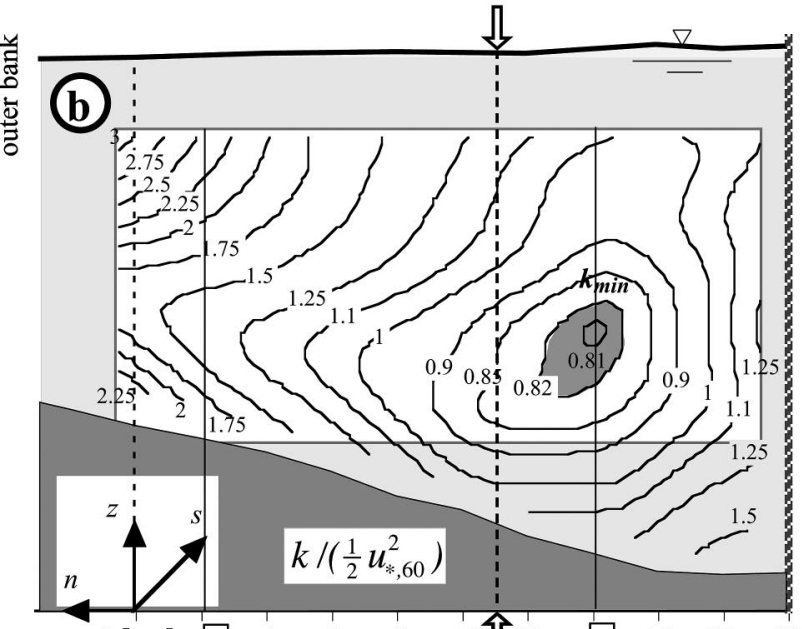

$0[\mathrm{~cm}] \quad-6 \begin{array}{llllllll}-4 & -6 & -8 & -10 \Uparrow-12 & \mathbf{E} & -16 & -18 & -20\end{array}$

(d)

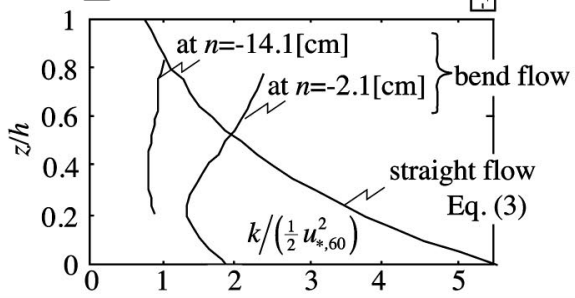

$K=\frac{1}{2}\left(v_{s}^{2}+v_{n}^{2}+v_{z}^{2}\right)$ and $k=\frac{1}{2}\left(\overline{v_{s}^{\prime 2}}+\overline{v_{n}^{\prime 2}}+\overline{v_{z}^{\prime 2}}\right)$

FIG. 3. (a) Isolines of normalized mean-flow kinetic energy $K /\left(1 / 2 U^{2}\right)$; (b) isolines of normalized turbulent kinetic energy $k /\left(1 / 2 u_{*, 60}^{2}\right) ;($ c) depth-averaged normalized mean flow and kinetic energy, $\langle K\rangle /\left(1 / 2 U^{2}\right)$ and $\langle k\rangle /\left(1 / 2 u_{* 60}^{2}\right)$, and ratio $\langle k\rangle /\langle K\rangle$; the arrows refer to the scale axes left or right; (d) vertical profiles of $k /\left(1 / 2 u_{*, 60}^{2}\right)$ in straight flow and bend flow. 
the velocity $v_{s}$ is smaller than $U$ at the inside of the cross section. As the cross-stream motion is rather weak, this means that the energy content there is $K /\left(1 / 2 U^{2}\right) \ll 1$. The mean flow kinetic energy is thus concentrated in the outer part of the bend. The mechanisms leading to this $K$ distribution have been analyzed by Blanckaert and Graf. ${ }^{2}$

The distribution of $k$ [Fig. 3(b)] exhibits the opposite pattern, in the water column as well as over the width. The core of minimum $k$ values nearly coincides with that of maximum $K$ values, and positive/negative gradients of $k$ correspond to negative/positive gradients of $K$. The depthaveraged value $\langle k\rangle /\left(1 / 2 u_{*, 60}^{2}\right)$ decreases from a centerline value of 2.2 to minimum values of about 1 in the outer-bank region, only to increase in the region affected by bank friction. In Fig. 3(d), the vertical profile of $k_{\text {straight }} /\left(1 / 2 u_{*, 60}^{2}\right)$ for straight uniform flow-according to Eq. (3) and based on $u_{*}=U \sqrt{C_{f}}$ with $C_{f} \approx 0.008$ - is compared with the vertical profiles of $k /\left(1 / 2 u_{*, 60}^{2}\right)$ measured at $n=-14.1 \mathrm{~cm}$ and $n=-2.1 \mathrm{~cm}$. In contrast to straight uniform flow, the measured profiles decrease from the bed to a minimum and then increase towards a maximum near the water surface [Fig. 3(b)]. Similar vertical $k$ profiles have been measured in channel bends and meanders by Tamai and Ikeya, ${ }^{15}$ Anwar, ${ }^{16}$ Muto, ${ }^{17}$ and Sudo et al. ${ }^{18}$

While in straight uniform flow the ratio $\langle k\rangle /\langle K\rangle$ would be constant (about 0.03) in the two-dimensional (2D) flow zone away from the banks, the opposite patterns of $K$ and $k$ in our experiment result in a pronounced variation of $\langle k\rangle /\langle K\rangle$ over the cross-section. Towards the centerline, $\langle k\rangle /\langle K\rangle$ is of the expected order of magnitude, but it then decreases, down to 0.01 in most of the outer bend, only to increase strongly in the region affected by bank friction. Note that the uncertainty in the depth-averaged values increases somewhat towards the outer bank, due to the extrapolations outside the measuring grid. This uncertainty, however, is too small to explain the difference between the expected values of $\langle k\rangle /\langle K\rangle \approx 0.03$ and the measured one of $O(0.01)$, and does not alter our conclusions.

In the next sections, we will investigate the mechanisms underlying the observed distributions of $k$ and $\langle k\rangle /\langle K\rangle$, especially in the outer bend.

\section{ANALYSIS}

\section{A. Transport equation for $k$}

The mechanisms underlying the distribution of tke are now investigated by means of a term-by-term analysis of the transport equation for $k$ (Hinze, ${ }^{19}$ see Batchelor ${ }^{20}$ for the transformation to curvilinear coordinates):

$$
\begin{aligned}
0=\frac{\partial k}{\partial t}= & -\left(\frac{1}{1+n / R} v_{s} \frac{\partial k}{\partial s}+v_{n} \frac{\partial k}{\partial n}+v_{z} \frac{\partial k}{\partial z}\right) \\
& -\left\{\frac{1}{1+n / R} \frac{\partial}{\partial s}\left[\overline{\left(\frac{p^{\prime}}{\rho}+k_{t}\right) v_{s}^{\prime}}\right]+\frac{1}{1+n / R} \frac{\partial}{\partial n}\left[(1+n / R) \overline{\left(\frac{p^{\prime}}{\rho}+k_{t}\right) v_{n}^{\prime}}\right]+\frac{\partial}{\partial z}\left[\overline{\left(\frac{p^{\prime}}{\rho}+k_{t}\right) v_{z}^{\prime}}\right]\right\}
\end{aligned}
$$

pressure-gradient work + turbulent $k$ transport

$$
\begin{aligned}
& -\left\{\left(\overline{v_{s}^{\prime 2}}-\frac{2}{3} k\right) e_{s s}+\left(\overline{v_{n}^{\prime 2}}-\frac{2}{3} k\right) e_{n n}+\left(\overline{v_{z}^{\prime 2}}-\frac{2}{3} k\right) e_{z z}+2 \overline{v_{s}^{\prime} v_{n}^{\prime}} e_{s n}+2 \overline{v_{s}^{\prime} v_{z}^{\prime}} e_{s z}+2 \overline{v_{n}^{\prime} v_{z}^{\prime}} e_{n z}\right\} \\
& \mathcal{P}=\text { production of } k=\text { exchange of kinetic energy between mean flow and turbulence } \\
& -\varepsilon(\nu)
\end{aligned}
$$

viscous dissipation of $k$,

where $k_{t}=1 / 2\left(v_{s}^{\prime 2}+v_{n}^{\prime 2}+v_{z}^{\prime 2}\right)$ denotes the instantaneous turbulent kinetic energy. Since they are not relevant to our experimental analysis, all terms containing the molecular viscosity $\nu$ are regrouped in $\varepsilon(\nu)$. Besides the viscous dissipation of turbulence it also contains some viscous diffusion, ${ }^{19}$ which is assumed to be negligibly small, given the high Reynolds number in these experiments. In Eq. (5), $t$ denotes time, $(1+n / R)$ is a metric factor accounting for the divergence of the radial coordinate axes, $p^{\prime}$ are the turbulent pressure fluctuations and $e_{i j}(i, j=s, n, z)$ are the strain rates, with $e_{s s}+e_{n n}+e_{z z}=0$.

The bracketed terms in the third line of Eq. (5), denoted by $\mathcal{P}$, are found with the opposite sign in the transport equation for the mean flow kinetic energy $K .{ }^{19}$ They represent the exchange of kinetic energy between mean flow and turbulence, which occurs through work of deformation of the mean motion by the turbulent stresses. Although negative contributions may exist in some regions of the flow domain 

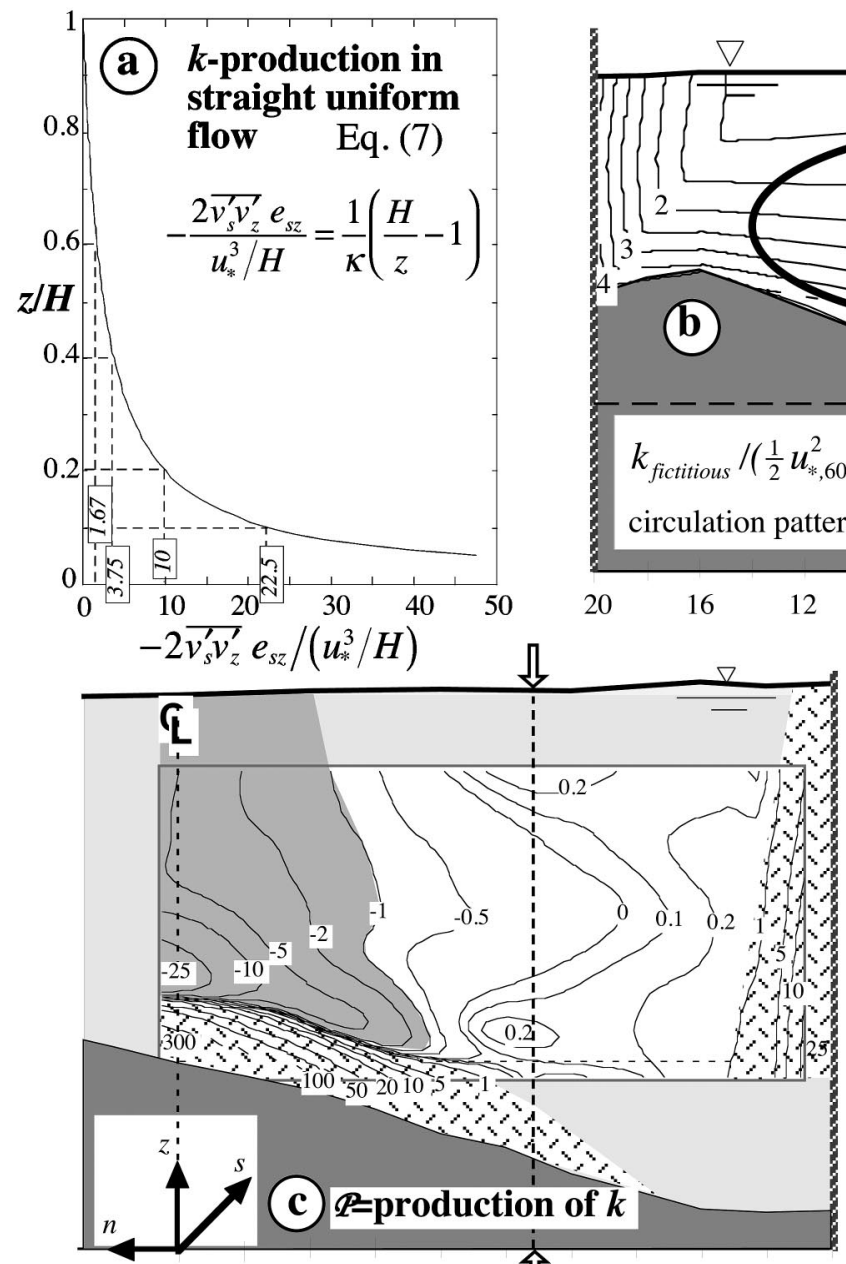

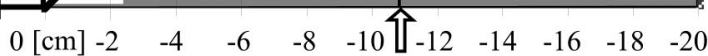
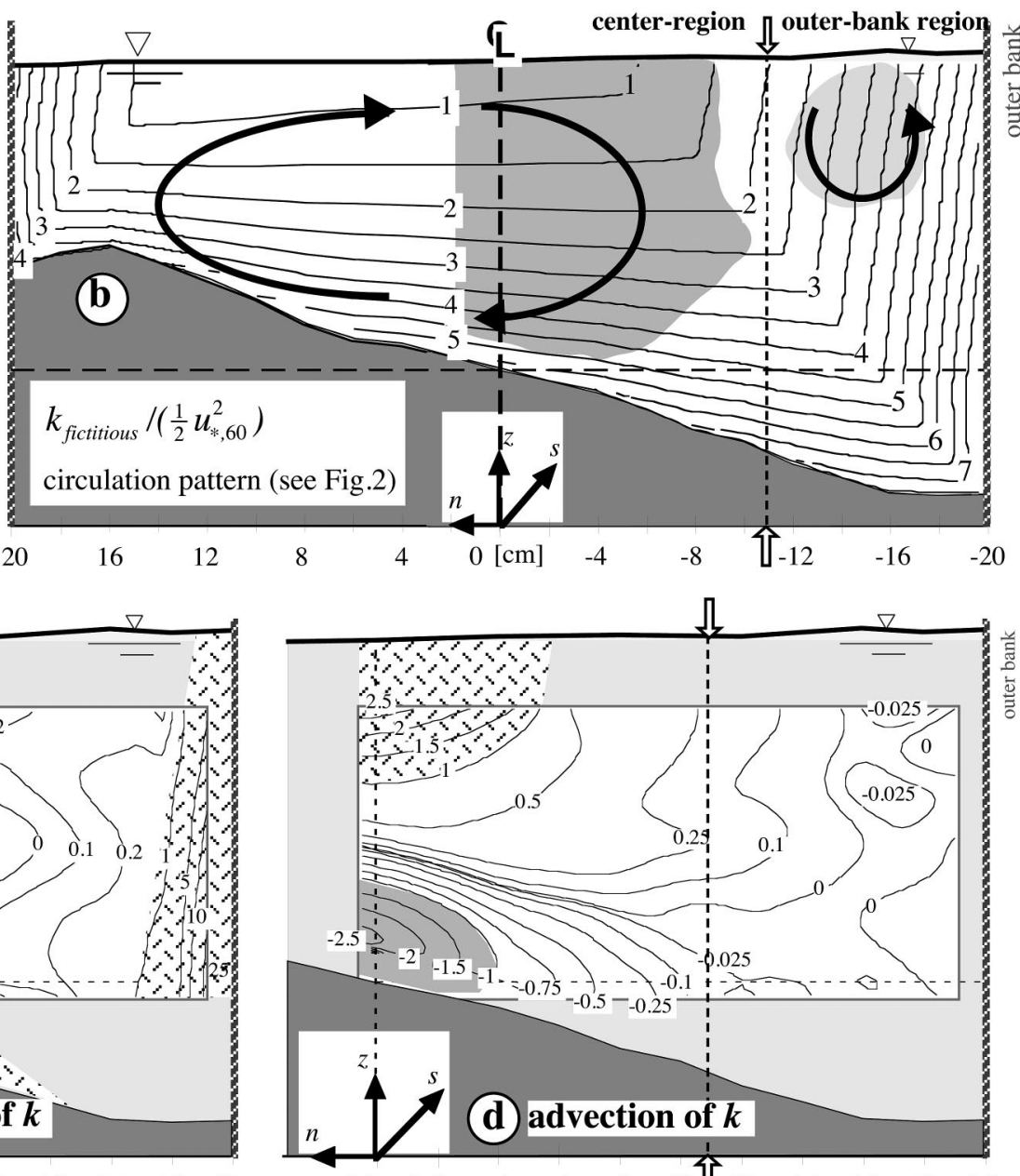

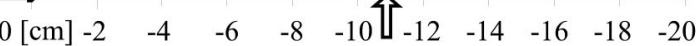

FIG. 4. (a) Normalized production of $k$ in straight uniform flow; (b) fictitious $k$ distribution as would exist in the absence of cross-stream motion and schematic pattern of cross-stream motion $\left(v_{n}, v_{z}\right)$ (cf. Fig. 2); (c) normalized production of $k$ measured in the experiment [cf. Eq. (5)]; (d) normalized advective transport of $k$ measured in the experiment [cf. Eq. (5)].

[also see Fig. 4(c)], the sum is globally positive and is commonly called the production of $k$. Globally speaking, $\mathcal{P}$ is balanced by the viscous dissipation $\varepsilon(v)$. Locally, $\mathcal{P}$ and $\varepsilon(\nu)$ may not balance, due to the transport terms in the first and second lines of Eq. (5). These terms do not generate or dissipate energy, but merely redistribute it from one point in the flow to another. ${ }^{21}$ The terms in the first line represent the advective transport of $k$ by the cross-stream motion $\left(v_{n}, v_{z}\right)$ and due to the flow non-uniformity $(\partial / \partial s)$, whereas the terms in the second line represent turbulent diffusion of $k$ and work done by the pressure gradient. Our experiment concerns steady flow $(\partial k / \partial t=0)$, but the unsteady term is retained in Eq. (5) to facilitate interpretation: positive/negative terms in the right-hand side correspond to terms that tend to increase/ decrease the local $k$.

The various terms in Eq. (5) are evaluated, as far as possible, from our experimental data, in order to gain insight into the mechanisms underlying the observed $k$ distribution. By definition, the terms related to downstream variations in the flow field $(\partial / \partial s$ terms; including those appearing implicitly in the strain rates $e_{s s}, e_{s n}$, and $e_{s z}$ ) cannot be evaluated from velocity measurements in a single cross section. The terms related to the pressure fluctuations could not be evaluated since the pressure was not measured. Knowledge of these terms would add to the understanding of curved-flow turbulence, but is not essential for the purpose of the present paper.

In straight uniform flow, neither cross-stream motion nor downstream or transversal gradients exist; therefore, Eq. (5) reduces to

$$
0=-\frac{\partial}{\partial z}\left(\overline{\left(\frac{p^{\prime}}{\rho}+k_{t}\right) v_{z}^{\prime}}\right)-2 \overline{v_{s}^{\prime} v_{z}^{\prime}} e_{s z}-\varepsilon(\nu) .
$$

Assuming a triangular distribution of $-\overline{v_{s}^{\prime} v_{z}^{\prime}}$ with values zero at the water surface and $u_{*}^{2}$ at the bed, and assuming a logarithmic vertical profile of the downstream velocity $v_{s}$ [Eq. (2)], one can easily derive that

$$
\begin{aligned}
& \mathcal{P}_{\text {straight }}=-2 \overline{v_{s}^{\prime} v_{z}^{\prime}} e_{s z}=\frac{1}{\kappa} \frac{u_{*}^{3}}{H}\left(\frac{H}{z}-1\right), \\
& \frac{-2 \overline{v_{s}^{\prime} v_{z}^{\prime}} e_{s z}}{u_{*}^{3} / H}=\frac{1}{\kappa}\left(\frac{H}{z}-1\right) .
\end{aligned}
$$


This function is shown in Fig. 4(a). The $k$-production is maximum at the bed and rapidly decreases towards the water surface. Clearly, bed friction is the principal source of tke. This justifies normalizing the evaluated terms in the $k$-equation by $u_{*, 60}^{3} / H=0.0008 \mathrm{~m}^{2} / \mathrm{s}^{3}$. The tke is redistributed over the flow depth by the first terms in Eq. (6) and dissipated by the last term.

Intuitively, one would expect the observed $k$ pattern in our curved-flow experiment to be redistributed (advective transport) by the cross-stream motion $\left(v_{n}, v_{z}\right)$. This is difficult to demonstrate, since the $k$ distribution strongly interacts with the cross-stream motion. In order to have an idea of its influence, we start from a fictitious $k$ distribution, as would exist in the absence of cross-stream motion, with high $k$ values near the flow boundaries and a rapid decrease away from those boundaries. Such a distribution, inspired by Eq. (3), is proportional to the bed shear stress $\tau_{b} / \rho=u_{*}^{2}$, which in the absence of advective momentum transport and neglecting influences from the banks, can be obtained from the simplified depth-integrated downstream momentum equation: ${ }^{2}$

$$
\frac{\tau_{b}}{\rho}=u_{*}^{2}=-\frac{1}{1+n / R} g h \frac{\partial z_{S}}{\partial s} .
$$

Here $\partial z_{s} / \partial s$ is nearly constant over the width of the bend; however, the physical water surface slope, (1 $+n / R)^{-1} \partial z_{s} / \partial s$, is larger in the inner bend than in the outer bend due to the metric factor $1+n / R(n / R>/<0$ in outer/ inner bend). This is the so-called potential-vortex effect that impels the locus of high velocity towards the inner bank. Over a natural bed topography, however, the influence of the local flow depth $h$ is dominant and $\tau_{b} / \rho=u_{*}^{2}$ increases in outward direction. The fictitious $k$ distribution shown in Fig. 4(b) is obtained by inserting the measured values of $\partial z_{s} / \partial s$ and $h$ in Eqs. (8) and (3). To account in a simple way for turbulence generation by bank friction, the fictitious $k$ pattern is taken symmetrical about the bisectors of the lower corners of the flow domain.

We now let the measured $\left(v_{n}, v_{z}\right)$-pattern act upon this fictitious $k$ distribution [Fig. 4(b)]. The center-region cell advects tke clockwise: the high near-bed $k$ values are concentrated in the inner bend; the high $k$ values near the inner bank spread out near the water surface in the inner bend; the low $k$ values near the water-surface shift towards the downward flow outer part of the center-region cell and give rise to a core of low $k$ values further down in the water column; the relatively low $k$ values originating from the region of downward flow between the two cells concentrate near the bed in the outer bend. Similarly, the outer-bank cell advects tke counterclockwise: the high $k$ values near the outer bank shift towards the water surface near the outer bank; the low values near the water surface shift towards the region where the outer-bank cell goes downwards and contributes to the core of low $k$ values; the relatively low $k$ values in the lower half of the outer-bank cell are advected toward the outer bank.

Qualitatively, the above description agrees well with the measured distribution of $k$ [see Fig. 3(b)].

Yet, the measured distribution of the advective $k$ transport by the cross-stream motion [Fig. 4(d)] seems not to support the above analysis. In the center region, the measured terms in Eq. (5) representing advective $k$ transport [Fig. 4(d)] are negative near the bed and thus tends to decrease the observed $k$, whereas it is positive near the water surface and tends to increase the observed $k$. Averaged over the flow depth, the positive and the negative contributions nearly compensate each other. In the outer-bank region, the measured advective $k$ transport is negligible. The measured advective $k$ transport can still explain the deformation of the vertical $k$ profiles as compared to the straight-uniform flow profiles, but not the observed distribution of $k$ over the width, especially the reduced values in the outer-bank region.

This apparent contradiction is due to the nonlinearity of the $k$ dynamics. Similar to the fictitious $k$ distribution, a fictitious $\mathcal{P}$ distribution can be assumed that would exist in the absence of cross-stream motion. Proportional to the local (shear) velocity cubed, it would increase in outward direction and have the same vertical profiles as in straight uniform flow [Eq. (7)]. Under the influence of the cross-stream motion, the fictitious $k$ distribution gradually modifies as described above, which results in a gradual redistribution of $\mathcal{P}$ and of the advective $k$-transport terms themselves.

The measured distribution of the production $\mathcal{P}$ [see Fig. 4(c)] strongly deviates from the fictitious distribution, over the flow depth as well as over the channel width. Whereas the fictitious $\mathcal{P}$ distribution increases in outward direction, the measured $\mathcal{P}$ distribution decreases. In the center region, $\mathcal{P}$ [Fig. 4(c)] is mainly due to bed friction and the measured $\mathcal{P}$ values are of the same order of magnitude as the typical near-bed values in straight uniform flow [Fig. 4(b)]. The near-bed values, however, should be interpreted with care, since the underlying velocity measurements are less reliable there. ${ }^{1}$ Contrary to the straight uniform flow profiles, negative values of $\mathcal{P}$ are observed over most of the flow depth, indicating a restitution of kinetic energy from the turbulence to the mean flow. These negative values, which reach normalized magnitudes as large as $O(-25)$, are almost entirely due to the $\overline{v_{s}^{\prime} v_{z}^{\prime}} e_{s z}$-contribution [Fig. 7(a) in Blanckaert and de Vriend $\left.{ }^{1}\right]$. Obviously, compared to a situation with only production of turbulent kinetic energy, i.e., $\mathcal{P}>0$, the existence of zones with $\mathcal{P}<0$ tends to reduce $k$. In the outerbank region, the magnitude of $\mathcal{P}$ is less than in the center region, which is mainly due to the negligible values of the turbulent shear stress $\overline{v_{s}^{\prime} v_{z}^{\prime}}$ [Fig. 6(a) in Blanckaert and $\mathrm{Graf}^{5}$ ] and of the corresponding $\overline{v_{s}^{\prime} v_{z}^{\prime}} e_{s z}$-contribution [Fig. 7 (a) in Blanckaert and de Vriend $\left.{ }^{1}\right]$ on the measuring grid. Values increase to $O(20)$ in the region affected by friction at the outer bank, indicating that the outer-bank friction is less than the bed friction in the center region, which leads to $\mathcal{P}$ values $O(300)$. This is in agreement with the measured distributions of $\overline{v_{s}^{\prime} v_{n}^{\prime}}$ and $\overline{v_{s}^{\prime} v_{z}^{\prime}}$ (Fig. 6 in Blanckaert and Graf ${ }^{5}$ ). The small outer-bank friction is explained partially by the smoothness of the outer bank as compared to the rough sand bed and partially by the reduced turbulence activity in the outer-bank region.

The turbulent diffusion in transversal direction (not shown) is found to be relatively small, with normalized magnitudes less than $O(0.1)$. The accuracy of the evaluated vertical diffusion terms (not shown) is poor. As expected, the 

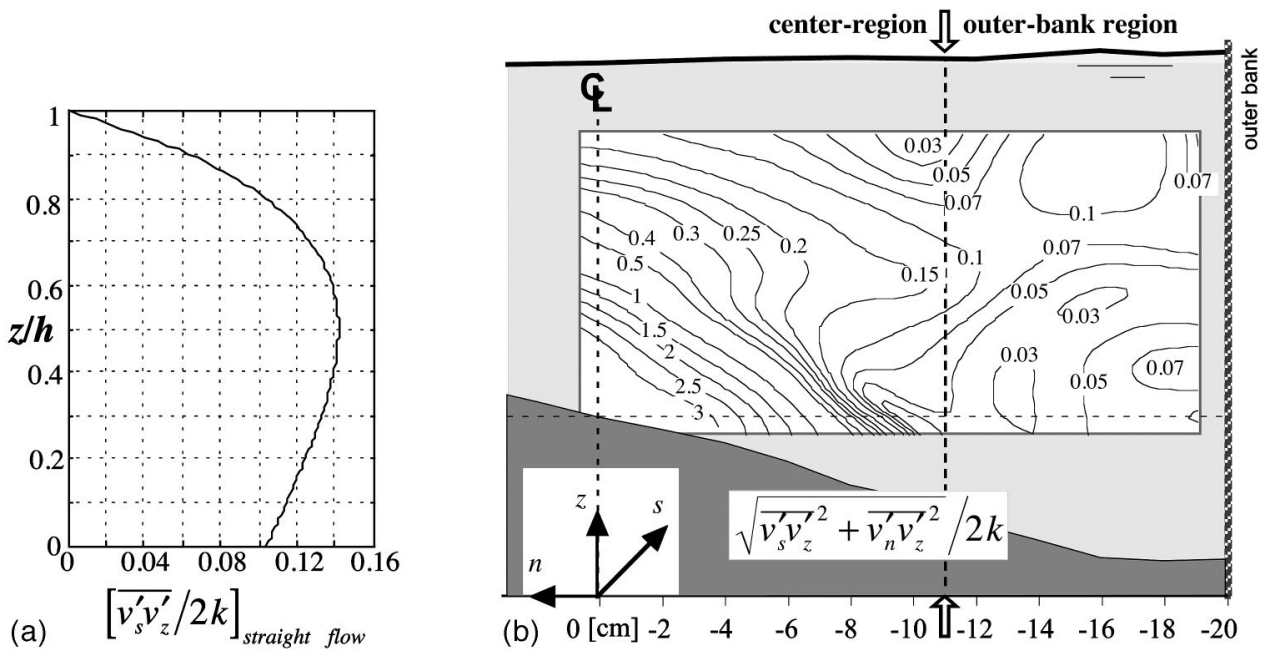

FIG. 5. Turbulence structure parameter $a_{1}=\sqrt{{\overline{v_{s}^{\prime} v_{z}^{\prime}}}^{2}+{\overline{v_{n}^{\prime} v_{z}^{2}}}^{2}} / 2 k$; (a) theoretical profile in straight uniform flow; (b) measured distribution in curved flow.

terms are positive near the bed, with relatively high normalized values of $O(50)$, and negative over most of the water column. By no means, these terms can explain the observed transversal distribution of $k$.

The above analysis leads to the following hypothesis about the mechanisms responsible of the observed distribution of $k$. In the absence of cross-stream motion, the fictitious production $\mathcal{P}$ increases in outward direction and leads to an outward increasing fictitious $k$ distribution as shown in Fig. 4(b). The advective $k$ transport by the cross-stream motion is at the origin of the redistribution of $k$. It tends to increase the $k$ values in the inner half of the cross section and to decrease them in the outer half. As $k$ gets redistributed, the advective $k$ transport becomes less efficient and ultimately, it does not explain the observed width distribution of $k$ anymore. However, as $k$ becomes redistributed also the production terms $\mathcal{P}$ are modified (the values of the turbulent stresses $\overline{v_{i}^{\prime} v_{j}^{\prime}}$ obviously depend on the available turbulent kinetic energy $k$ ). Whereas the initial fictitious $\mathcal{P}$ increases in outward direction, the modified $\mathcal{P}$ distribution ultimately decreases in outward direction. It can be concluded that this ultimate $\mathcal{P}$ distribution is the major factor responsible of the measured outward decrease of $k$. Especially the low values of the $\overline{v_{s}^{\prime} v_{z}^{\prime}} e_{s z}$ contribution in the outer-bank region might be important for the reduced $k$ values.

Obviously there is a strong feedback between the reduced levels of $k$ and the low values of the production term $\mathcal{P}$ in the outer-bank region. From the distributions of $k$ and of $\mathcal{P}$, however, it is not clear to what extent the reduced $k$ is caused by the low $\mathcal{P}$ values, or conversely. This issue will be investigated by considering some typical turbulence characteristics in Sec. IV B and discussed in Sec. IV C.

\section{B. Turbulence structure}

In the previous chapter, the causal relation between $k$ and $\mathcal{P}$ did not become clear. We will therefore take a closer look at the turbulence-structure parameters $a_{1}$ and $\nu_{j k}$ (the mixing coefficients). The role of these turbulence-structure parameters in the $k-\mathcal{P}$ relationship and the $k$ distribution will be discussed in Sec. IV C. A rather extensive presentation of the mixing coefficients is justified by the fact that they are also important to the spreading and mixing of heat and dissolved or suspended matter (sediments, pollutants, oxygen, etc.).

\section{Structure parameter $a_{1}$}

An important turbulence-structure parameter is $a_{1}$, defined as ${ }^{22,23}$

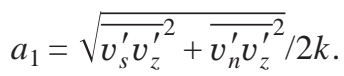

According to Schwarz and Bradshaw, ${ }^{22}$ it can be regarded as a first indicator of the efficiency of turbulent eddies in producing shear, given the amount of tke. The parameter $a_{1}$ is typically larger in two dimensional than in three-dimensional flows. These smaller values of $a_{1}$ in various types of threedimensional flows are shown and physically explained by Schwarz and Bradshaw ${ }^{22}$ and Piquet. ${ }^{23}$ To our knowledge, values of $a_{1}$ for three-dimensional open-channel flow have not been reported before.

The vertical profile of $a_{1}$ for straight uniform openchannel flow is shown in Fig. 5(a). It is based on a triangular profile of $-\overline{v_{s}^{\prime} v_{z}^{\prime}}$ that increases from 0 at the water surface to $u_{*}^{2}$ at the bed, under the assumption that $\overline{v_{n}^{\prime} v_{z}^{\prime}}=0$ and that Eq. (3) holds for $k$. The $a_{1}$ profile increases from 0 at the water surface, reaches a maximum of about 0.14 around mid-depth and subsequently decreases to about 0.1 at the bed. The experimental distribution of $a_{1}$ for our curved open-channel flow is shown in Fig. 5(b). In the center region, values are high, $O(1)$, mainly due to very high values of the $s-z$ component of the Reynolds stress, which are probably associated with the deformation of the downstream velocity profile and the downward directed secondary flow. ${ }^{24}$ Moreover, the distribution of $k$ in this area does not exhibit the sharp increase near the bed which is common in straight uniform flow. Finally, it has to be noted that the measured velocities are not very accurate in the lowest $20 \%$ of the water column; ${ }^{1}$ therefore the values may be overestimated there. Outward from 
the centerline, a strong reduction of $a_{1}$ is observed: $a_{1}$ $=O(0.1)$ near the separation of the two circulation cells and even less in the outer-bank region. This strong outward decrease of $a_{1}$ corresponds to a similar outward decrease of $\overline{v_{s}^{\prime} v_{z}^{\prime}} 5$. Apparently, the efficiency of shear production decreases towards the outer bend.

\section{Mixing coefficients}

The mixing coefficients $\nu_{j k}$ also tell something about the turbulence structure. Similar to the definition of the eddy viscosity, they are defined as the ratio between the deviatoric turbulent stresses $-\left(\overline{v_{j}^{\prime} v_{k}^{\prime}}-2 / 3 \delta_{j k} k\right)$ and the corresponding strain rates $e_{j k}$ :

$$
\nu_{j k}=\nu_{k j}=-\frac{\overline{v_{j}^{\prime} v_{k}^{\prime}}-2 / 3 \delta_{j k} k}{2 e_{j k}}(j, k=s, n, z),
$$

where $\delta_{j k}$ is the Kronecker delta. Note that these six mixing coefficients do not have the frame-indifferent characteristics of the Reynolds stress tensor $\overline{v_{j}^{\prime} v_{k}^{\prime}}$ or the strain rate tensor $e_{j k}$. Applying the definition of the mixing coefficients, the terms representing production of turbulent kinetic energy in Eq. (5) can be rewritten as

$\mathcal{P}=2\left(\nu_{s s} e_{s s}^{2}+\nu_{n n} e_{n n}^{2}+\nu_{z z} e_{z z}^{2}+2 \nu_{s n} e_{s n}^{2}+2 \nu_{s z} e_{s z}^{2}+2 \nu_{n z} e_{n z}^{2}\right)$.

According to Eqs. (10) and (11a), the mixing coefficients can be regarded as indicators of the efficiency of the strain rates in producing turbulence. The sign of the mixing coefficient $\nu_{j k}$ corresponds to the sign of the energy exchange term $-\left(v_{j}^{\prime} v_{k}^{\prime}-2 / 3 \delta_{j k} k\right) e_{j k}$ in $\mathcal{P}$. $\nu_{j k}>0$ corresponds to production of tke, whereas $\nu_{j k}<0$ corresponds to restitution of kinetic energy from the turbulence to the mean flow via the corresponding turbulent stress $\overline{v_{j}^{\prime} v_{k}^{\prime}}$.

The mixing coefficients are particularly important with respect to environmental problems such as the spreading and mixing of heat and suspended or dissolved matter. Those phenomena are described by an advection-diffusion equation, in which the diffusion coefficients (Rutherford ${ }^{25}$ ) are commonly taken proportional to the mixing coefficients.

In numerical models, often a scalar eddy viscosity is adopted for turbulence closure, which implies that $\nu_{s s}=\nu_{n n}$ $=\nu_{z z}=\nu_{s n}=\nu_{s z}=\nu_{n z}=\nu_{t}>0$ and that $\mathcal{P}$ is definitely positive:

$$
\mathcal{P}=2 \nu_{t}\left(e_{s s}^{2}+e_{n n}^{2}+e_{z z}^{2}+2 e_{s n}^{2}+2 e_{s z}^{2}+2 e_{n z}^{2}\right)>0 \text {. }
$$

In straight uniform flow, it is common practice to prescribe a parabolic distribution of $\nu_{t}$ over the water column, with zero values at the bed and the water surface and a depth-averaged value of $\left\langle\nu_{t}\right\rangle_{\text {straight }}=0.067 u * h$. This profile corresponds to the logarithmic downstream velocity profile. The experimental mixing coefficients shown in Fig. 6 are normalized by this reference value for straight uniform flow, $\left\langle\nu_{t}\right\rangle_{\text {straight }}$ $=0.067 u_{*, 60} H$.

The distributions of all mixing coefficients shown in Figs. 6(a)-6(e) contain positive as well as negative values. Near the bed, mainly positive values are found, whereas negative values occur mainly in the upper part of the water column. The latter correspond to a restitution of kinetic en- ergy from the turbulence to the mean flow via the turbulent stress $\overline{v_{j}^{\prime} v_{k}^{\prime}}$. Obviously, the existence of zones with negative mixing coefficients tends to reduce $k$ with respect to the situation in which there is only production of turbulent kinetic energy $\left(v_{j k}>0\right)$. Negative values of the mixing coefficients $v_{j k}$ - or of the corresponding kinetic energy exchange $-\left(\overline{v_{j}^{\prime} v_{k}^{\prime}}-2 / 3 \delta_{j k} k\right) e_{j k}$-have been reported before. $^{16,26-28}$ Blanckaert and de Vriend $^{1}$ have shown that this restitution of kinetic energy from the turbulence to the mean flow plays an important role in the generation of the outer-bank cell of cross-stream circulation in the present experiment (cf. Fig. 2).

Looking at the distribution over the water column, the magnitude of the experimental mixing coefficients has a tendency to be minimal near the bed and near the water surface and to have a maximum somewhere halfway the water column. Qualitatively, this is in agreement with the parabolic profile used in straight uniform flow.

As stated before, the magnitudes (absolute values are indicated by \|\|$)$ of the mixing coefficients are very important to the modeling of spreading and mixing processes. However, experimental data on these mixing coefficients are scarce. The present data show that the coefficient $\left\|\nu_{n n}\right\|[$ Fig. $6(a)]$ is maximum near the centerline, decreases towards a minimum near the separator between the two circulation cells and increases again towards the outer bank. The coefficient $\left\|\nu_{z z}\right\|$ [Fig. 6(b)] shows the opposite behavior and has its maximum in the separation zone between the two cells. As mentioned before, the $(s, n)$ and the $(s, z)$ contributions are dominant in the production of tke. The magnitude of the corresponding mixing coefficients $\left\|\nu_{s z}\right\|$ and $\left\|\nu_{s n}\right\|$ [Figs. 6(c) and 6(d), respectively] strongly decreases in the outward direction, from $O(1$ to 10$)$ in the center region to $O(0.1$ to 1$)$ in the outer-bank region. $\left\|\nu_{n z}\right\|[$ Fig. 6(e)] behaves similarly, except that it assumes high values near the center of the outerbank cell. This outward decrease of the mixing coefficients related to the turbulent shear stresses is in agreement with the outward decrease of the coefficient $a_{1}$ [Fig. 5(b)] and confirms the observation that the efficiency of shear stress production for a given turbulent kinetic energy is reduced in curved flow.

Figure 6(f) shows the lateral distribution in the outer bend of the mixing coefficients $\left\langle\left\|\nu_{j k}\right\|\right\rangle$, being the depthaveraged absolute values evaluated within the measuring grid and excluding the asymptotic values where the strain tends to zero. These lateral distributions confirm the behavior observed in the three-dimensional distributions. Note the strong decrease of $\left\langle\left\|\nu_{s z}\right\|\right\rangle$ in outward direction, with very low values in the outer-bank region. $\left\langle\left\|\nu_{s n}\right\|\right\rangle$ reaches similar small values in the outer-bank region. The normalized average values over the entire measuring grid, $\left\langle\left\langle\left\|\nu_{j k}\right\|\right\rangle\right\rangle$, shown in the table below Fig. 6(f), are all $O(1)$. The dominant component is $(n, n)$, with a normalized value of 5.32 , which should be compared with the values of 1.5 (laboratory channels) to 6 (irregular waterways) reported by Graf and Altinakar. ${ }^{29}$ It is remarkable that the components related to the turbulent normal stresses are larger than those related to the turbulent shear stresses. 


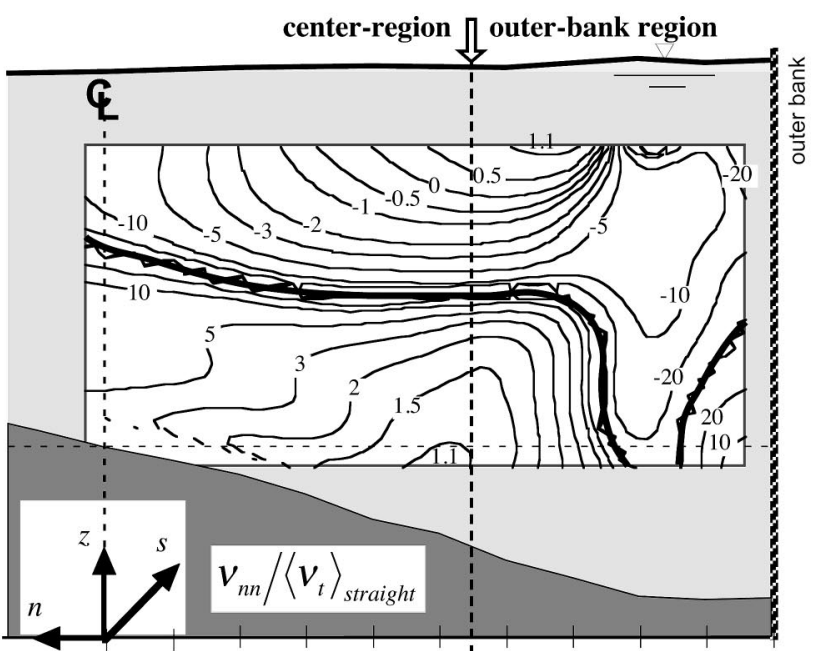

(a) $0[\mathrm{~cm}]-2 \quad$\begin{tabular}{lllll:lllll}
\hline & -4 & -6 & -8 & -10 & -12 & -14 & -16 & -18 & -20
\end{tabular}

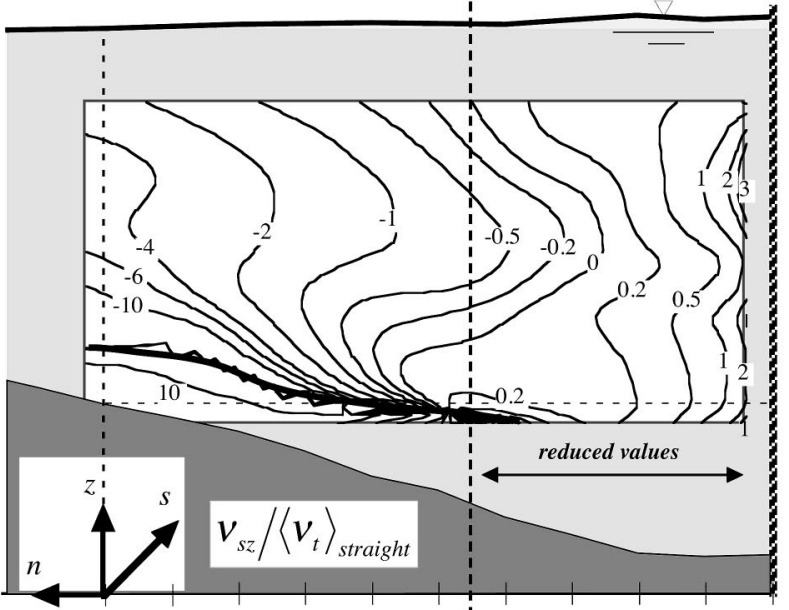

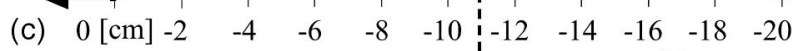

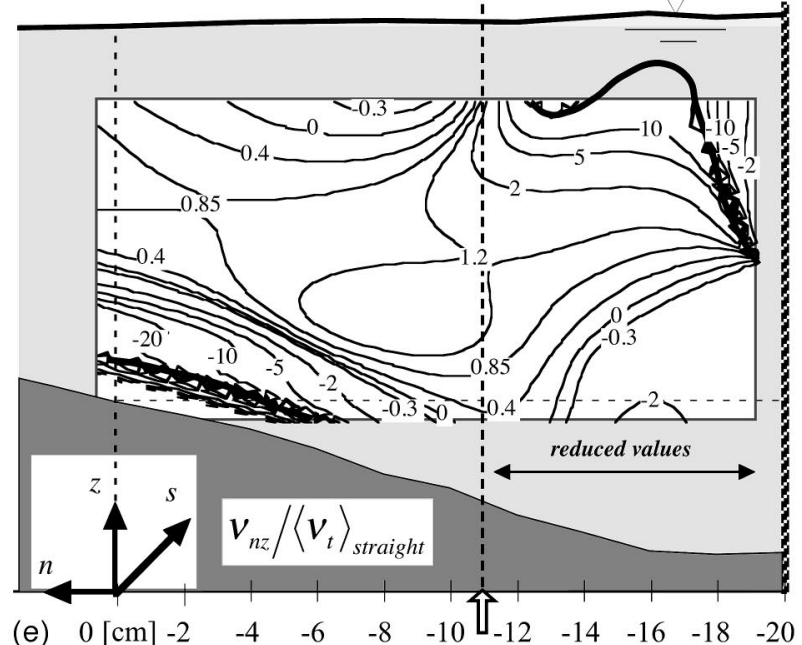

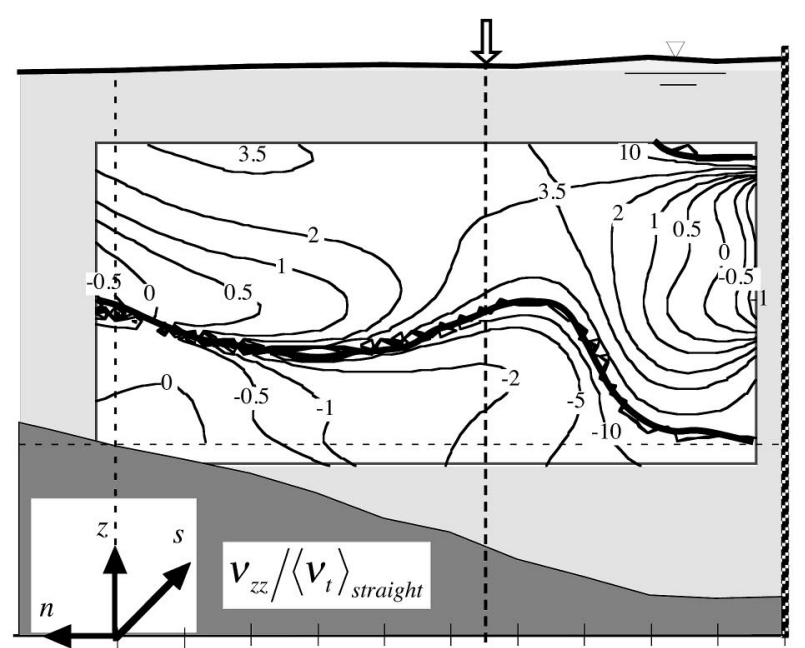

(b) $0[\mathrm{~cm}]-2 \quad \begin{array}{llll:lllll}-4 & -6 & -8 & -10 & -12 & -14 & -16 & -18 & -20\end{array}$

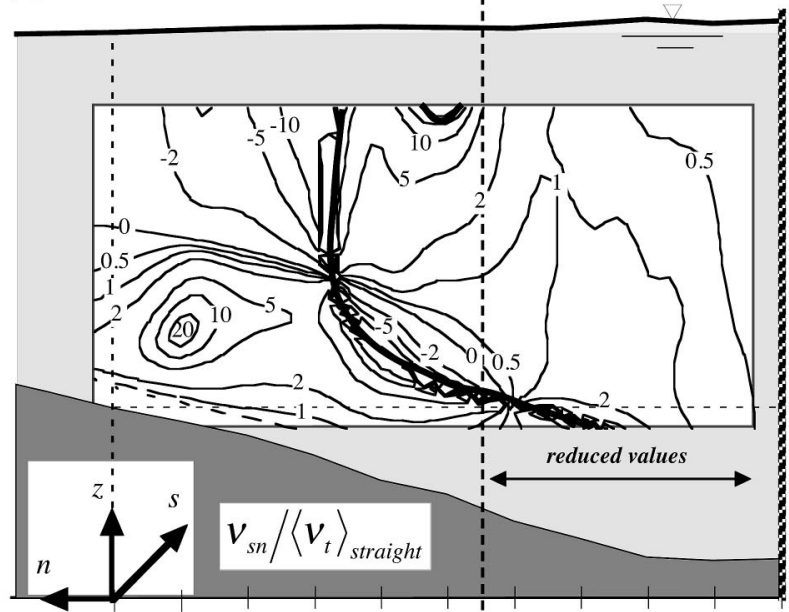

(d) $0[\mathrm{~cm}]-2 \quad \begin{array}{llll:lllll}-4 & -6 & -8 & -10 & -12 & -14 & -16 & -18 & -20\end{array}$

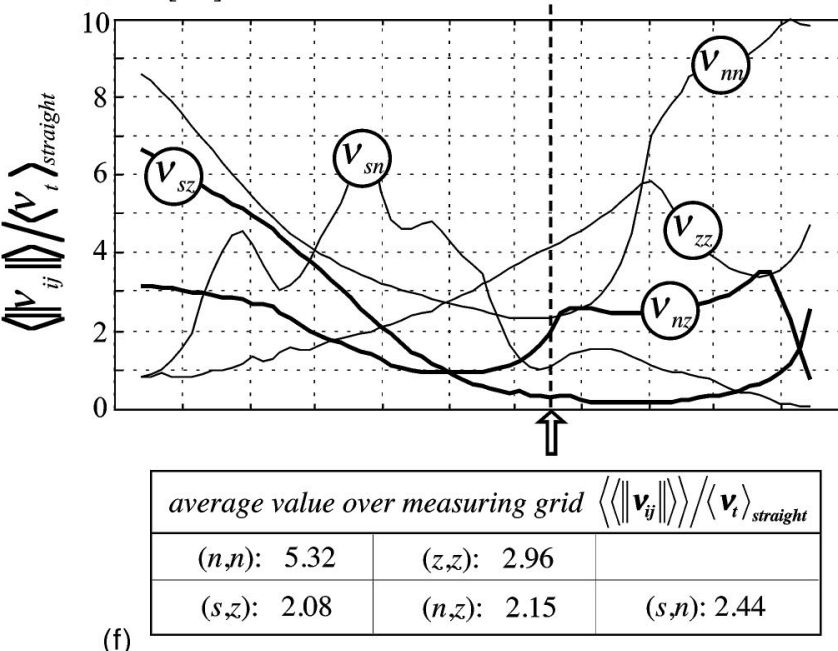

(f)

(10)]; (f) width distribution of depth-averaged mixing coefficients.

\section{Physical mechanisms}

The analysis of the transport equation for $k$ indicated that the reduced turbulence activity in the outer-bank region cannot be explained solely from the advective redistribution of tke by the cross-stream motion. This phenomenon is primarily due to a decrease of the tke production. The parameter $a_{1}$ and the mixing coefficients $\nu_{j k}(\mathrm{Sec}$. IV B) indicate that the turbulence structure in our curved flow experiment differs significantly from that in straight uniform flow. Given the same amount of turbulent kinetic energy $k$ the turbulent shear stresses $\overline{v_{j}^{\prime} v_{k}^{\prime}}(j \neq k)$ turn out to be significantly smaller. In other words, the Reynolds stress tensor is more diagonally dominant in curved flow.

The observed decrease of the turbulence activity in the 
outer-bank region can be explained from these findings as follows. Suppose we start from the turbulence structure in straight uniform flow. If less turbulent shear stress is produced while the $k$ level remains the same, this leads to a reduced production of tke [see Eq. (5)], and less production obviously results in lower $k$ levels. The lower $k$ levels, in their turn, lead to smaller turbulent stresses $\overline{v_{j}^{\prime} v_{k}^{\prime}}$ and thus also to a smaller production $\mathcal{P}$. Apparently, the feedback is negative, so that it ultimately leads to a reduced, but nonzero $k$ level.

Blanckaert and de Vriend ${ }^{30}$ have further analyzed the turbulence structure of the same flow field. They found that the velocity fluctuations are atypically coherent over the width and therefore decomposed them into slow coherent fluctuations and a background signal. The slow fluctuations represent a bulk oscillation of the pattern of circulation cells. A spectral analysis shows that the slow fluctuations have the characteristics of a wavelike motion (low efficiency of shear generation) whereas the background signal has the characteristics of developed turbulence. This explains in physical terms why the total velocity fluctuations lead to less shear stress production than if all of it were developed turbulence.

\section{MODELING IMPLICATIONS}

\section{A. Standard turbulence closure}

Consequently, for the accurate modeling of the $k$ distribution it is not sufficient that the turbulence closure includes the transport equation for $k$ [Eq. (5)], it also needs to accurately describe the turbulence structure, i.e., the distribution of the turbulence energy among the different turbulent normal stresses as well as the distribution of the turbulent shear stresses. Factors that influence the turbulence structure are the cross-stream motion, downstream and transversal pressure gradients, accelerations and decelerations along streamlines, the curvature of the streamlines, the relative bed roughness, etc. Large eddy simulation (LES) models or turbulence closures that use a transport equation for each of the turbulent stresses-such as Reynolds stress models-can account for all these factors. Such models are computationally expensive, which explains why lower-order turbulence closures are the most commonly used in practice. The widely used twoequation closures, however, do not account for the factors mentioned above. The $k-\varepsilon$ model, for instance, which is often used for open-channel flows, contains the transport equation for $k$, but uses a scalar eddy viscosity, which means that the deviatoric turbulent stresses, $-\left(\overline{v_{j}^{\prime} v_{k}^{\prime}}-2 / 3 \delta_{j k} k\right)$, are taken proportional to the strain rates, $e_{j k}$ [see Eqs. (10), (11a), and (11b)]. It cannot accurately represent the turbulence structure, since $\nu_{t}$ cannot account for the observed behavior of the mixing coefficients (cf. Fig. 6).

(i) $\quad v_{t}$ is definitely positive, whereas the experimental mixing coefficients have positive as well as negative values.

(ii) $\quad \nu_{t}$ is a scalar, whereas the six experimental mixing coefficients have different magnitudes and different distributions.

(iii) The scalar $\nu_{t}$ cannot account for the different behavior of the mixing coefficients related to the turbulent normal stresses and those related to the turbulent shear stresses; especially the reduction of the latter in the outer bend cannot be reproduced.

All factors making the turbulence structure in our experiment deviate from that in straight channel flow directly or indirectly result from the main flow curvature. Therefore, it seems worthwhile to attempt finding a relationship between the changes in the turbulence structure and a curvature parameter. Such a relationship may be useful to make semiempirical extensions to existing turbulence closure models, in order to improve their capabilities in curved open-channel flow.

\section{B. Stratification analogue}

\section{Two-dimensional shear flows curved in their own plane}

Theoretical and numerical ${ }^{31-36}$ as well as experimental $^{37-39}$ investigations for two-dimensional shear flows curved in their own plane have shown that the structure of turbulence is very sensitive to the streamline curvature. Its influence is an order of magnitude larger than predicted by straightforward extensions of calculation methods for simple shear layers.

Bradshaw ${ }^{40,41}$ has established a formal analogy between the influence of streamline curvature and the influence of buoyancy. While buoyancy leads to a density stratification of the flow, curvature leads to a pressure stratification. The analysis usually applied to derive buoyancy parameters from the equations of motion can be used to formally derive analogous curvature parameters. Extensions of standard turbulence closures based on such parameters have been proposed for a variety of two-dimensional shear flows, characterized by streamline curvature in the plane of main shear. $^{31,33-35,38}$

One such parameter is the curvature-flux-Richardson number, defined as

$$
\begin{aligned}
R_{f} & =\left(-2 \overline{v_{s}^{\prime} v_{n}^{\prime}} \frac{v_{s}}{r_{s n}}\right) /\left(-\overline{v_{s}^{\prime} v_{n}^{\prime}} \frac{\partial r_{s n} v_{s}}{\partial n}\right) \\
& =\frac{2 v_{s}}{r_{s n}} /\left(\frac{1}{r_{s n}} \frac{\partial r_{s n} v_{s}}{\partial n}\right) \approx \frac{2 v_{s}}{r_{s n}} /\left(\frac{\partial v_{s}}{\partial n}+\frac{v_{s}}{r_{s n}}\right),
\end{aligned}
$$

where $r_{s n}$ is the local radius of streamline curvature-which is negative for the investigated left-turning bend-and $n$ is the direction in which the flow is curved. $R_{f}$ is interpreted as the ratio of the curvature-induced production of $\left(-\overline{v_{n}^{\prime 2}}\right)$ to the total $\overline{v_{s}^{\prime 2}}$ production. In these two-dimensional flows curved in their own plane, turbulence is damped if $R_{f}>0$ and enhanced if $R_{f}<0$. Furthermore, the distribution of the turbulent kinetic energy among the turbulent normal stresses is altered: $\overline{v_{n}^{\prime 2}} / \overline{v_{s}^{\prime 2}}$ decreases as $R_{f}>0$ and increases as $R_{f}<0$.

\section{Three-dimensional open-channel flows curved in the horizontal plane}

Although our experiment concerns a more complex three-dimensional flow field, curved in a different plane and 


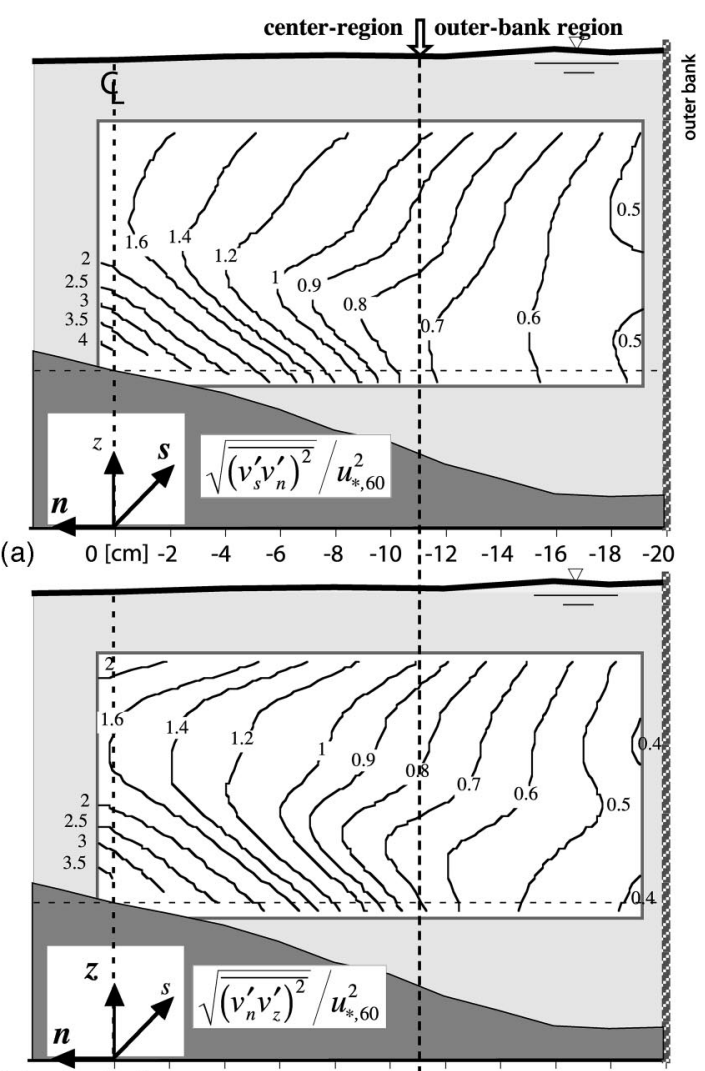

(c)
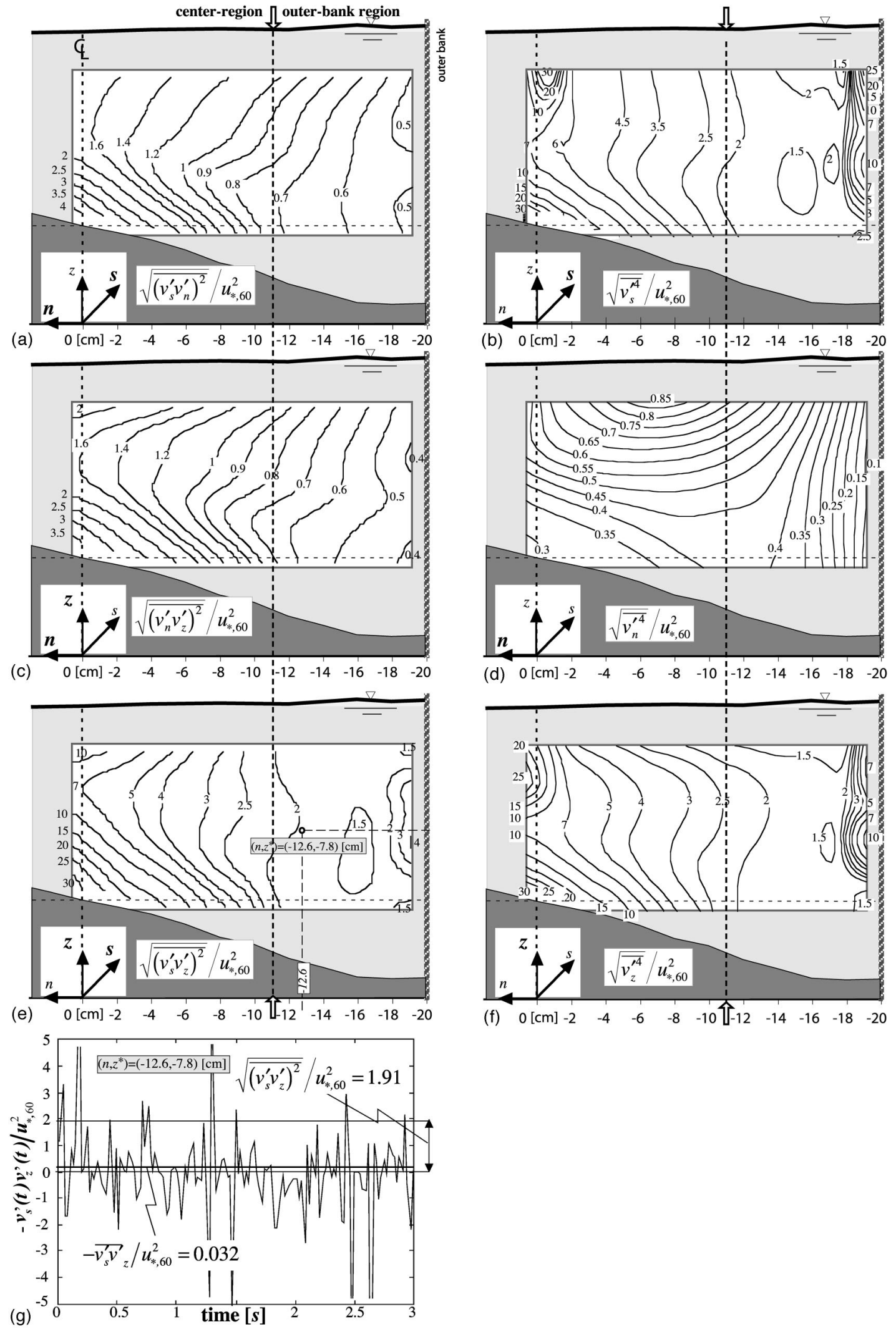

FIG. 7. (a)-(f) Isolines of normalized fourth-order turbulent correlations $\sqrt{\left(v_{i}^{\prime} v_{j}^{\prime}\right)^{2}} / u_{*, 60}^{2}$; (g) time series of $v_{s}^{\prime}(t) v_{z}^{\prime}(t) / u_{* 60}^{2}$ at the point $\left(n, z^{*}\right)=(-12.6$, $-7.8)(\mathrm{cm})$ 
with the strong cross-stream motion as the main complicating factor, the flow field seems to be stratified in the plane of curvature. This is most obvious from the square root of the fourth-order turbulent moments, $\sqrt{\left(v_{j}^{\prime} v_{k}^{\prime}\right)^{2}}$, shown in Figs. 7(a)-7(f) normalized by $u_{*, 60}^{2}$.

With the exception of the $(n, n)$ component, all components $\sqrt{\overline{\left(v_{j}^{\prime} v_{k}^{\prime}\right)^{2}}}$ are rather uniformly distributed over the water column and decrease rather strongly in the outward direction, in compliance with the reduced turbulence activity towards the outer bank. These higher-order turbulence characteristics suggest that the analogy between buoyancy stratification and curvature stratification also holds in complex three-dimensional curved flows.

These fourth-order turbulent moments characterize the magnitude of the instantaneous values of the quantities $v_{j}^{\prime}(t) v_{k}^{\prime}(t)$. These instantaneous values are at least as important as their time-averaged values $\overline{v_{j}^{\prime} v_{k}^{\prime}}$, being the turbulent stresses, if it comes to the spreading and mixing of heat and dissolved or suspended matter, sediment transport, and erosion of alluvial boundaries. In regions of low turbulent stress, considerable positive and negative instantaneous values can be reached. This is illustrated for the turbulent shear stress $\overline{v_{s}^{\prime} v_{z}^{\prime}}$ at $\left(n, z^{*}\right)=(-12.6,-7.8) \mathrm{cm}$, in Fig. $7(\mathrm{~g})\left(z^{*}\right.$ denotes the distance below the water surface).

Demuren and $\operatorname{Rodi}^{42}$ and Ye and McCorquodale ${ }^{43}$ have applied a curvature extension to the $k$ - $\varepsilon$ model for the simulation of flow and pollutant dispersion in open-channels curved in the horizontal plane. They found that the inclusion of curvature effects can significantly affect the results and adopted a modified version of the curvature extension proposed by Leschziner and Rodi ${ }^{33}$ for two-dimensional shear flows curved in their own plane:

$$
\begin{aligned}
\nu_{t}=c_{\mu} \frac{k^{2}}{\varepsilon} \quad \text { with } \quad c_{\mu} & =\frac{0.09}{1+0.57 \frac{k^{2}}{\varepsilon^{2}}\left(\frac{\partial v_{s}}{\partial n}+\frac{v_{s}}{r_{s n}}\right) \frac{v_{s}}{r_{s n}}} \\
& =\frac{0.09}{1+0.57 \frac{k^{2}}{\varepsilon^{2}}\left(\frac{\partial v_{s}}{\partial n}\right)^{2} \frac{2 R_{f}}{\left(2-R_{f}\right)^{2}}} .
\end{aligned}
$$

Generally speaking, such semiempirical extensions will not be good enough to correctly represent the turbulence structure in highly three-dimensional flows with a significant cross-stream motion. In order to be useful, they need to be based on a large amount of experimental data. In the following, we will investigate to what extent the turbulence structure in our case of three-dimensional curved open-channel flow can be related to the curvature-flux-Richardson number.

\section{Experimental observations}

Figure 8 compares the transversal distributions of the depth-averaged curvature-flux-Richardson number $\left\langle R_{f}\right\rangle$ and the turbulence activity $\langle k\rangle /\langle K\rangle$. More details can be found in Blanckaert $^{44}$ (Chap. III 3).

There seems to be a rather strong relationship between the two. Near the centerline, where $\left\langle R_{f}\right\rangle \sim 0,\langle k\rangle /\langle K\rangle$ ap-

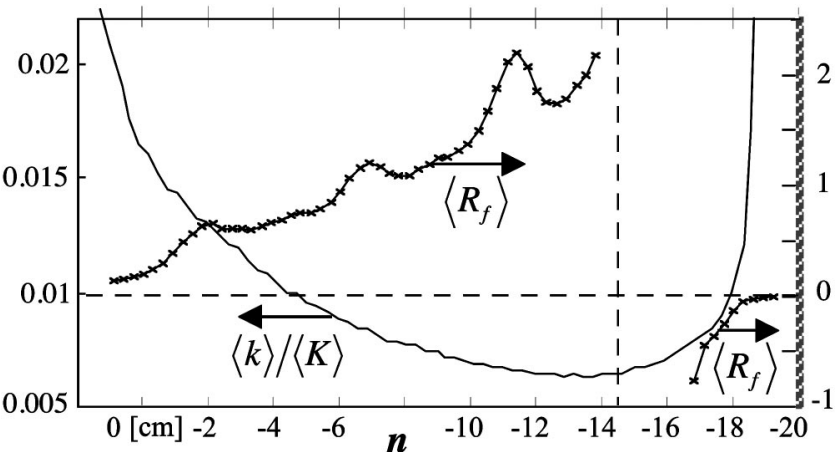

FIG. 8. Depth-averaged flux-curvature-Richardson number $\left\langle R_{f}\right\rangle$, and ratio $\langle k\rangle /\langle K\rangle$ [cf. Fig. 3(c)]. The arrows refer to the scale axes left or right.

proaches its straight-uniform-flow value [cf. Eq. (4)]. The outward decrease of $\langle k\rangle /\langle K\rangle$ is accompanied by increasing positive values of $\left\langle R_{f}\right\rangle$; the location of the maximum $\left\langle R_{f}\right\rangle$ coincides with that of the minimum $\langle k\rangle /\langle K\rangle$. In the outerbank shear layer, the influence of the bank proximity dominates the influence of the streamline curvature: $\left\langle R_{f}\right\rangle$ is negative and $\langle k\rangle /\langle K\rangle$ strongly increases.

Figures 9(a) and 9(b) show the distributions of $\overline{v_{n}^{\prime 2}} / \overline{v_{s}^{\prime 2}}$ and $\overline{v_{z}^{\prime 2}} / \overline{v_{s}^{\prime 2}}$, representative of the distribution of the turbulent kinetic energy $k$ among the turbulent normal stresses. Distributions of all the turbulent stresses have been presented in Blanckaert and Graf. $5 \overline{v_{s}^{\prime 2}}$ and $\overline{v_{z}^{\prime 2}}$ have rather similar distributions over the width, as appears from the nearly horizontal isolines. The smaller values of $\overline{v_{z}^{\prime 2}} / \overline{v_{s}^{\prime 2}}$ near the bed and near the water surface are due to the geometrical constraint on the vertical fluctuations. The ratio $\overline{v_{n}^{\prime 2}} / \overline{v_{s}^{\prime 2}}$ is rather uniform over the water column, but has a pronounced transversal distribution, which is due to the almost opposite behavior of $\overline{v_{s}^{\prime 2}}$ and $\overline{v_{n}^{\prime 2}}$. The values of these ratios averaged over the entire measuring grid, $\langle\langle\rangle\rangle$,

$$
\left\langle\left\langle\overline{v_{n}^{\prime 2}} / \overline{v_{s}^{\prime 2}}\right\rangle\right\rangle=0.34\left\langle\left\langle\left\langle\overline{v_{z}^{\prime 2}} / \overline{v_{s}^{\prime 2}}\right\rangle\right\rangle=0.47,\right.
$$

show that the ratio $\overline{v_{n}^{\prime 2}} / \overline{v_{s}^{\prime 2}}$ is smaller and $\overline{v_{z}^{\prime 2}} / \overline{v_{s}^{\prime 2}}$ is larger in the curved flow experiment than in their counterpart in straight uniform flow, where these ratios are nearly constant $\mathrm{at}^{14}$

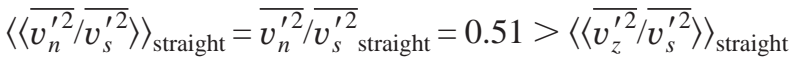

$$
\begin{aligned}
& =\overline{v_{z}^{\prime 2}} \overline{v_{s}^{\prime 2} \text { straight }}=0.31 \text {. }
\end{aligned}
$$

In line with the expectations from the theoretical considerations on 2D shear flows curved in their own plane, our observations indicate that the curvature has a stabilizing effect for $\left\langle R_{f}\right\rangle>0$ and leads to smaller values of the ratio $\overline{v_{n}^{\prime 2}} / \overline{v_{s}^{\prime 2}}$, that seem to be compensated by larger values of $\overline{v_{z}^{\prime 2}} / \overline{v_{s}^{\prime 2}}$. The stabilizing curvature seems to affect the transversal structure of the flow field $\left(R_{f}\right.$ and $\overline{v_{n}^{\prime 2}} / \overline{v_{s}^{\prime 2}}$ distributions), but much less the vertical structure.

\section{SUMMARY AND CONCLUSIONS}

This paper reports on an experimental investigation of turbulence characteristics in a sharp open-channel bend. This 

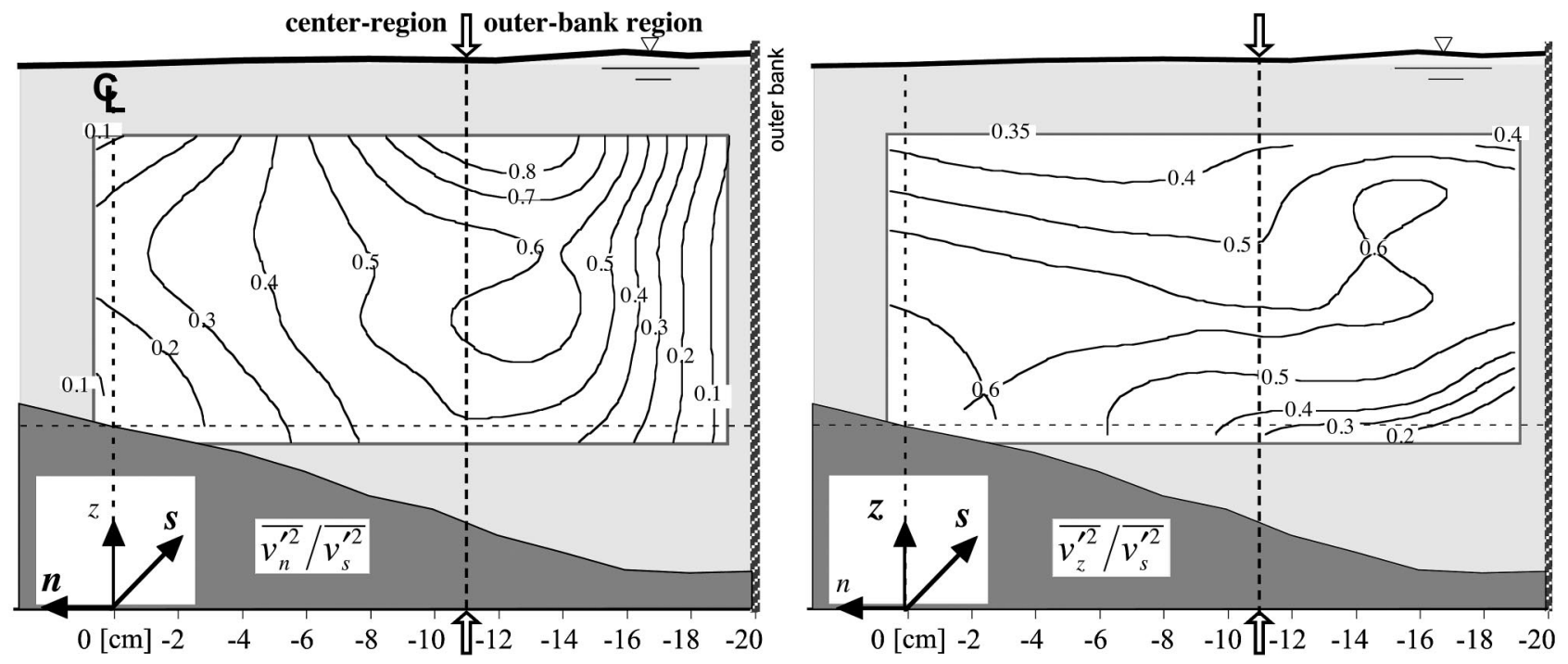

FIG. 9. Ratios of the turbulent normal stresses: (a) $\overline{v_{n}^{\prime 2}} / \overline{v_{s}^{\prime 2}}$; (b) $\overline{v_{z}^{\prime 2}} / \overline{v_{s}^{\prime 2}}$.

fills a gap in the availability of turbulence data that has hampered the modeling of such flows, so far.

In the outer half of one cross section of a laboratory open-channel bend, three-dimensional velocity measurements were made with an acoustic Doppler velocity profiler (ADVP) on a fine grid. This instrument takes simultaneous high-resolution measurements of all three velocity components in a horizontal line coinciding with the instrument's axis. From these measurements, the mean velocity vector was derived, as well as the fluctuating velocity vector, all six turbulent stress components and all higher-order turbulent velocity correlations. Typical of the flow in the investigated cross section are the existence of a bicellular pattern of crossstream circulation and a significantly reduced turbulence activity in the outer bend.

The observed distributions of all six turbulent stress components have been reported elsewhere. ${ }^{5}$ In the present paper, distributions of various other turbulence characteristics are given, such as the turbulent kinetic energy, the ratio between the depth-averaged turbulent kinetic energy and the depth-averaged mean flow kinetic energy, the turbulencestructure parameter $a_{1}$, the mixing coefficients, the ratios between the turbulent normal stresses, and the square roots of the fourth-order turbulent correlations.

An analysis of the mechanisms leading to the reduced turbulence activity in the outer bend was made, via a termby-term evaluation of the transport equation for the turbulent kinetic energy $k$ on the basis of the measured data. Special attention was given to the terms representing the production of turbulent kinetic energy and those representing its advective transport by the cross-stream motion. The rather speculative conclusion is that there is a negative feedback between transport and production. The advective transport redistributes $k$ over the cross section and thereby modifies the distribution of the production $\mathcal{P}$, hence the turbulence structure, hence $k$. In the ultimate $k$ distribution the influence of the cross-stream motion has almost vanished and it is mainly the modified $\mathcal{P}$ distribution that is responsible for the observed reduction of $k$ towards the outer bank.

The relationship between the turbulent kinetic energy and its production is complex and characterized by a feedback mechanism. It is shown to depend mainly on the turbulence structure, represented by the structure parameter $a_{1}$ and the mixing coefficients. The turbulence structure in our experiment is found to differ significantly from that in straight uniform flow. Both $a_{1}$ and the mixing coefficients indicate that the efficiency of shear stress production for a given amount of turbulent kinetic energy is less in curved-channel flow than in straight uniform flow (the Reynolds stress tensor is more diagonally dominant), and that it decreases towards the outer bank. This modified turbulence structure explains the observed reduction of turbulence activity in the outer bend.

All departures from the turbulence structure in straight uniform flow are directly or indirectly due to the imposed streamline curvature. Bradshaw ${ }^{40,41}$ has established a formal analogy between streamline curvature and buoyancy in twodimensional shear layer flows curved in their own plane. The relevant control parameter is the curvature-flux-Richardson number $R_{f}$. The turbulence structure in our case of highly three-dimensional flow, which is curved in the horizontal plane, appears to be related to $R_{f}$, as well. The departures from straight uniform flow qualitatively agree with what could be expected on the basis of this analogy with twodimensional curved flow: turbulence is reduced for $R_{f}>0$ and the ratio $\overline{v_{n}^{\prime 2}} / \overline{v_{s}^{\prime 2}}$ is decreased. The influence of the streamline curvature acts primarily in the horizontal and brings about a kind of transversal stratification in the turbulence structure. The latter is further confirmed by the distributions of the fourth-order turbulent correlations.

Consequently, it is not sufficient for turbulence closure models to incorporate the transport equation for the turbulent kinetic energy: they also have to represent the turbulence 
structure. Two-equation turbulence models, such as the commonly used $k-\varepsilon$ model, are inherently unable to do so. Semiempirical extensions to two-equation turbulence closures based on the curvature-flux-Richardson $R_{f}$ number, as proposed for two-dimensional curved flows, may be used to improve the applicability of such closures to threedimensional curved flows. This requires more experimental data, however, with a larger spatial coverage and for a wider range of hydraulic and geometric conditions.

It should be emphasized that our experimental data originate from a single cross section at $60^{\circ}$ from the bend entrance, under one set of hydraulic $(\mathrm{Fr}, C)$ and geometric $(R / B, B / H)$ conditions. The experimental program is presently extended to more cross sections around the bend and a wider range of hydraulic conditions in a larger and less narrow flume. ${ }^{44}$

\section{ACKNOWLEDGMENTS}

This research was sponsored by the Swiss National Science Foundation under Grant Nos. 2100-052257.97/1 and 2000-059392.99/2. The first author acknowledges his Ph.D. supervisor W. H. Graf, as well as Professor Zech and Professor Booij for their review of the manuscript.

${ }^{1}$ K. Blanckaert and H. J. de Vriend, "Secondary flow in sharp open-channel bends," J. Fluid Mech. 498, 353 (2004).

${ }^{2}$ K. Blanckaert and W. H. Graf, "Momentum transport in sharp openchannel bends," J. Hydraul. Eng. 130, 186 (2004).

${ }^{3}$ A. J. Odgaard, "Bank erosion contribution to stream sediment load," Iowa Institute of Hydraulic Research, Iowa, Report No. 280, 1984.

${ }^{4}$ W. E. Dietrich, "Mechanics of flow and sediment transport in river bends," River Channels: Environment and Process, edited by K. Richards (Inst. Brit. Geogr. spec., Oxford, 1987), pp. 179-227.

${ }^{5} \mathrm{~K}$. Blanckaert and W. H. Graf, "Experiments on flow in an open-channel bend. Mean flow and turbulence," J. Hydraul. Eng. 127, 835 (2001).

${ }^{6}$ D. Hurther, "3-D acoustic Doppler velocimetry and turbulence in openchannel flow," Ph.D. thesis Nr 2395, Ecole Polytechnique Fédérale Lausanne, Switzerland, 2001.

${ }^{7}$ T. Rolland, "Développement d'une instrumentation Doppler ultrasonore: application aux écoulements turbulents en hydraulique," $\mathrm{Ph} . \mathrm{D}$. thesis $\mathrm{Nr}$ 1281, Ecole Polytechnique Fédérale Lausanne, Switzerland, 1994.

${ }^{8}$ U. Lemmin and T. Rolland, "Acoustic velocity profiler for laboratory and field studies," J. Hydraul. Eng. 123, 1089 (1997).

${ }^{9}$ D. Hurther and U. Lemmin, "A constant beamwidth transducer for threedimensional Doppler profile measurements in open channel flow," Meas. Sci. Technol. 9, 1706 (1998).

${ }^{10}$ D. Hurther and U. Lemmin, "A correction method for turbulence measurements with a 3-D acoustic Doppler velocity profiler," J. Atmos. Ocean. Technol. 18, 446 (2001).

${ }^{11} \mathrm{~K}$. Blanckaert and U. Lemmin, "Means of noise reduction in acoustic turbulence measurements," J. Hydraul. Res. (to be published).

${ }^{12}$ C. de Boor, A Practical Guide to Splines (Springer, Berlin, 1978).

${ }^{13} \mathrm{~K}$. Blanckaert and H. J. de Vriend, "Non-linear modeling of mean flow redistribution in curved open channels," Water Resour. Res. 39, 1375 (2003).

${ }^{14}$ I. Nezu and H. Nakagawa, Turbulence in Open-Channel Flows (IAHRmonograph, Balkema, 1993), p. 54.

${ }^{15} \mathrm{~N}$. Tamai and T. Ikeya, "Three-dimensional flow over alternating point bars in a meandering channel," J. Hydrosci. Hydr. Eng. 3, 1 (1985).
${ }^{16}$ H. O. Anwar, "Turbulent structure in a river bend," J. Hydraul. Eng. 112, 657 (1986)

${ }^{17}$ Y. Muto, "Turbulent flow in two-stage meandering channels," Ph.D. thesis, Bradford University, Bradford, UK, 1997.

${ }^{18}$ K. Sudo, M. Sumida, and H. Hibara, "Experimental investigation on turbulent flow in a square-sectioned 90-degree bend," Exp. Fluids 30, 246 (2001).

${ }^{19}$ J. O. Hinze, Turbulence (McGraw-Hill, New York, 1975).

${ }^{20}$ G. K. Batchelor, An Introduction to Fluid Dynamics (Cambridge University Press, Cambridge, 1970).

${ }^{21}$ H. Tennekes and J. L. Lumley, A First Course in Turbulence (MIT, Cambridge, MA, 1972).

${ }^{22}$ W. R. Schwarz and P. Bradshaw, "Turbulence structural changes for a three-dimensional turbulent boundary layer in a $30^{\circ}$ bend," J. Fluid Mech. 272, 183 (1994)

${ }^{23}$ J. Piquet, Turbulent Flows: Models and Physics (Springer, Berlin, 1999).

${ }^{24}$ I. Nezu, H. Nakagawa, and A. Tominaga, "Secondary currents in a straight channel flow and the relation to its aspect ratio," Turbulent Shear Flows 4, edited by L. J. S. Bradbury et al. (Springer, Berlin, 1985), pp. 246-260.

${ }^{25}$ J. C. Rutherford, River Mixing (Wiley, Chichester, UK, 1994), p. 33.

${ }^{26}$ R. Booij and J. Tukker, "3-dimensional laser-Doppler measurements in a curved flume," Developments in Laser Techniques and Applications to Fluid Mechanics (Springer, Berlin, 1996), pp. 98-114.

${ }^{27} \mathrm{~K}$. Shiono and Y. Muto, "Complex flow mechanisms in compound meandering channels with overbank flow," J. Fluid Mech. 376, 221 (1998).

${ }^{28} \mathrm{R}$. Booij, "Measurements and large eddy simulations of the flows in some curved flumes," J. Turbul. 4, 1 (2003).

${ }^{29}$ W. H. Graf and M. Altinakar, Fluvial Hydraulics (Wiley, Chichester, UK, 1998).

${ }^{30} \mathrm{~K}$. Blanckaert and H. J. de Vriend, "Turbulence structure in sharp openchannel bends," J. Fluid Mech. (to be published).

${ }^{31}$ H. P. A. H. Irwin and P. A. Smith, "Prediction of the effect of streamline curvature on turbulence," Phys. Fluids 18, 624 (1975).

${ }^{32}$ M. M. Gibson and W. Rodi, "A Reynolds-stress closure model of turbulence applied to the calculation of a highly curved mixing layer," J. Fluid Mech. 103, 161 (1981).

${ }^{33}$ M. A. Leschziner and W. Rodi, "Calculation of annular and twin parallel jets using various discretization schemes and turbulence-model variations," ASME Trans. J. Fluids Eng. 103, 352 (1981).

${ }^{34}$ W. Rodi and G. Scheuerer, "Calculation of curved shear layers with twoequation turbulence models," Phys. Fluids 26, 1422 (1983).

${ }^{35}$ G. C. Cheng and S. Farokhi, "On turbulent flows dominated by curvature effects," ASME Trans. J. Fluids Eng. 114, 52 (1992).

${ }^{36}$ A. G. L. Holloway and S. Tavoularis, "Geometric explanation of the effects of mild streamline curvature on the turbulence anisotropy," Phys. Fluids 10, 1733 (1998).

${ }^{37}$ R. M. C. So and G. L. Mellor, "Experiment on convex curvature effects in turbulent boundary layers," J. Fluid Mech. 60, 43 (1973).

${ }^{38}$ A. G. L. Holloway and S. Tavoularis, "The effects of curvature on sheared turbulence," J. Fluid Mech. 237, 569 (1992).

${ }^{39}$ B. Chebbi, A. G. L. Holloway, and S. Tavoularis, "The response of sheared turbulence to changes in curvature," J. Fluid Mech. 358, 223 (1998).

${ }^{40} \mathrm{P}$. Bradshaw, "The analogy between streamline curvature and buoyancy in turbulent shear flow,” J. Fluid Mech. 36, 177 (1969).

${ }^{41} \mathrm{P}$. Bradshaw, "Effects of streamline curvature on turbulent flow," AGARDograph 169 (1973).

${ }^{42}$ A. O. Demuren and W. Rodi, "Calculation of flow and pollutant dispersion in meandering channels," J. Fluid Mech. 172, 63 (1986).

${ }^{43}$ J. Ye and J. A. McCorquodale, "Simulation of curved open channel flow by 3D hydrodynamic model," J. Hydraul. Eng. 124, 687 (1998).

${ }^{44} \mathrm{~K}$. Blanckaert, "Flow and turbulence in sharp open-channel bends," Ph.D. thesis, Nr 2545, Ecole Polytechnique Fédérale Lausanne, Switzerland (available on ftp://lrhmac15.epfl.ch/Pub/Thesis/Blanckaert/PhD) (2002). 